\title{
Modeling of Macro Fiber Composite actuated laminate plates and aerofoils
}

Journal Title

$\mathrm{XX}(\mathrm{X}): 1-27$

(C) The Author(s) 2016

Reprints and permission:

sagepub.co.uk/journalsPermissions.nav

DOI: 10.1177/ToBeAssigned

www.sagepub.com/

\section{Peter R Thomas, Ángela Carmen Blázquez Calzada, and Kevin Gilmour}

\begin{abstract}
This paper investigates the modeling of Macro Fiber Composite (MFC)-actuated laminate plates with distributed actuator patches. The investigation details an analytical and finite element modeling, with experimental validation of the bending strain and deflection of an epoxy E-glass fiber composite laminate. An analytical approach is also developed to estimate the plate deflection from the experimental strain measurements. The analytical method uses direct integration of single dimensional plate bending moments obtained by strain-induced shear moments from the MFC actuators. Finite element analysis software was used with the composite laminate modeled in ANSYS ACP. The results from both analytical and numerical models show good agreement with the experimental results, with strain values agreeing within $20 \mathrm{ppm}$ and the maximum difference in deflection not exceeding $0.1 \mathrm{~mm}$ between models. Lastly, an application of the analytical model for developing morphing aerofoil designs is demonstrated.
\end{abstract}

\section{Keywords}

Macro Fiber Composite, Composite Laminate, Finite Element Analysis, Morphing aerofoil,

\section{Introduction}

Within recent development of smart materials attention has been focused on developing novel actuation techniques for small unmanned vehicles. For example, the concept of morphing for unmanned air vehicles (UAVs) provides even more advantages such as a short time-to-deliver due to the specific and reduced certification and qualification tests for these vehicles, and an increase in the amount of morphing technologies, since UAVs are exposed to lower aerodynamic loads. For small UAVs, the conventional method is the employment of a servomechanism that moves a hinged control surface. However, the use of smart material actuators is becoming more frequent due to the advantages they provide in comparison to servomechanism systems such as simplicity, larger control bandwidth and the reduction of drag due to the lack of discontinuities on the aircraft surfaces (Probst 2012).

The most common types of smart materials are the ones that deal with the application of temperature that result in plastic deformation (shape memory alloys), the implementation of a magnetic field modifying the body shape (magnetostrictive), and the coupling of electric current and mechanical deformation (piezoelectrics). The principal advantage of piezoelectric materials is the immediate and precisely variation in the shape or the electrical field. Considerable research and development by NASA Langley Research Center (LaRC) resulting in a series of cost effective piezoelectric actuators, developed from the 1990s onwards (Figure 1). The THUNDER actuator was derived from the RAINBOW piezoelectric materials produced by Clemson University, increasing its motion along the vertical axis. The Macro Fiber Composite (MFC) comes from the Active fiber Composite (AFC) line, adding to it an increased component of unidirectional strain. Finally, Radial Field Diaphragm (RFD) evolved from MFC and THUNDER, including not only the high displacement in the vertical axis but a radial distribution of the electric field. Many of these materials are now available commercially. Some of the applications of these three actuators/sensors include piezoelectric motors, health monitoring, structural vibration, vibration suppression, acoustic transduction, computational modeling development, precision valve control and metering, synthetic

School of Engineering and Computer Science, University of Hertfordshire, UK

\section{Corresponding author:}

Peter Thomas, University of Hertfordshire, College Lane, Hatfield, Hertfordshire, AL10 9AB, UK.

Email: p.thomas5@herts.ac.uk 


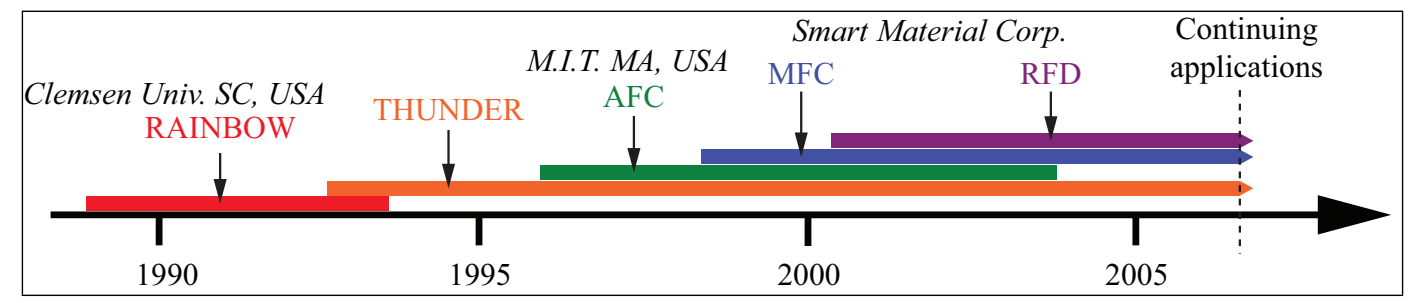

Figure 1. Timeline of post 1985 piezoelectric actuators showing commercial availability. Adapted from Bryant (2007).

jets, optical strain gauges, aerofoil shaping and energy harvesting (Bryant 2007).

\section{Macro Fiber Composite}

The MFC actuator was designed by NASA in 1996 and later was made commercially available from 2002. MFCs are constructed from rectangular PZT (lead zirconate titanate) fibers embedded in an epoxy matrix and sandwiched between two films of Kapton, on which the interdigitated electrodes are etched (Williams et al. 2002). The significant difference of MFCs over the previous generation AFCs is the use of rectangular fibers which allows for a greater fiber volume ratio in the composite layer. The low-cost manufacturing process of the fibers also helped to reduce the manufacturing cost and improved the commercial viability. The interdigitated pattern is responsible of carrying the applied voltage straight to and from the ribbon-shaped rods and consequently changing the shape of the structure to which it is embedded to (Williams et al. 2004). Due to its minimal thickness, the actuator is ideal for its use on thin structures, such as wings. On the other hand, if no voltage is applied, this piezoelectric material can behave as an extremely sensitive strain gauge able to measure deformations, noise and vibrations.

There are two types of MFC actuators shown in Figure 2:

1. A P1 type, where the polarisation is parallel to the piezo-fiber direction. This means that the induced strain is aligned to the electrodes in a perpendicular way.

2. A P2 type, where the polarisation is in the thickness direction.

The $\mathrm{P} 1$ and $\mathrm{P} 2$ types represent piezoelectric responses in different axes, effectively described as $d_{33}$ and $d_{31}$ effects, respectively. The $d_{33}$ effect is typically more powerful than the more traditional $d_{31}$.

Considerable work has been done at Virginia Polytechnic Institute and State University in characterising the behaviour of the MFCs (Schultz 2003; Lloyd 2004; Williams 2004; Williams et al. 2004). MFCs have been particularly effective as actuators in morphing surfaces and many researches have utilised them in the investigation and testing of morphing wings. A series of work by Bilgen et al. demonstrated basic roll and pitch control using MFC actuators to warp the composite wings of a small remote-controlled aircraft (Bilgen et al. 2009) and a bimorph aerofoil (Bilgen et al. 2010). Bilgen and Friswell then went on to implement optimisation techniques to control the actuation of MFC patches on a variable camber aerofoil (Bilgen and Friswell 2012, 2013). Dwarakanathan et al. (2015) modeled and collected wind tunnel data for a MFC-actuated hingeless flap on a glass fiber bimorph micro air vehicle (MAV). Gustafson (2011) designed, simulated, and verified with a wing tunnel, a morphing trailing edge of a wing in which a composite aerofoil was designed in a MFC bimorph configuration for a MAV. Ohanian III et al. (2012, 2013) compared an MFC-actuated MAV to a conventional flap actuated one, noting improved aerodynamic efficiency and response speed but at the expense of larger weight and overall reduced manoeuvrability. Molinari et al. (2014, 2015) also investigated roll control by numerical simulation, demonstrating the viability of roll control by actuation with MFCs. In 2013, Debiasi et al. (2013b,a) developed two variable camber model wings firstly with a compliant upper surface followed by both upper and lower surface actuation. They modeled the skin deflection for the first model with a functional geometrical model by relating the expansion in length of the unimorph to the resulting variation in curvature (Debiasi et al. 2013b). Experiments on the second, bimorph, model showed variations in lift coefficient of about \pm 0.1 and pitching moment coefficient of \pm 0.05 following changes to the wing camber (Debiasi et al. 2013a). More recently, Fichera et al. (2019) used a sandwich arrangements of MFCs to actuate the trailing edge, focusing on high bandwidth actuation that cannot be achieved with traditional servo motors.

\section{Modeling of MFCs}

General analytical models for piezoelectric composite structures have focused on simple unimorph and bimorph 


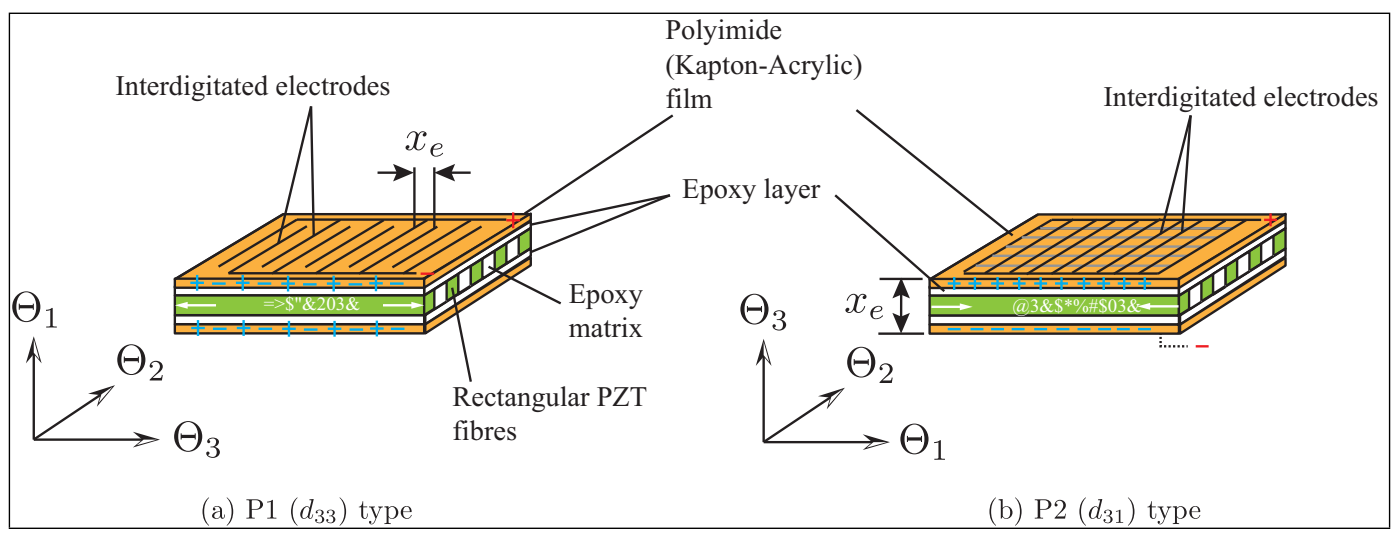

Figure 2. P1 and P2 type Macro fiber Composites.

cantilevers due to to the interest in designing efficient microelectro-mechanical sensors and actuators (DeVoe and Pisano 1997; Weinberg 1999; Li et al. 1999; Gao et al. 2009). Most of this is based on Euler-Bernoulli modeling of composite beams, similar to the original treatment of bi-metallic beam equations of Stoney (1909) and Timoshenko (1925) but specifically derived for piezoelectric-actuated structures (Crawley and Anderson 1990). An alternative modeling technique, whereby each lamina is modeled individually is referred to as the 'pin-force' model (Crawley and DeLuis 1987). This has its usefulness in that the strain in each lamina can be modeled separately. It is generally not as accurate as the Euler-Bernoulli approach but has seen subsequent improvements in the modeling accuracy in recent years. In particular, Li et al. (2016) present a recent development of this approach and specifically with reference to MFCactuated structures.

Most other investigations of MFC-actuated structures have made use of finite element (FE) modeling due to the greater flexibility afforded by the techniques and availability of commercial FE programs. Chee et al. (1998) provide a succinct summary of the origins of FEA work on modeling piezoelectric materials. Although some popular FEA packages such as ANSYS and ABAQUS have provided mechanisms to model the piezoelectric effect these were not always straightforward to use and were not implemented to use data in the same format as the ANSI/IEEE standards (Reaves and Horta 2001). MSC/NASTRAN, despite being as widely used, did not posses an inherent modeling approach for piezoelectricity which led to the development of an alternative thermal strain analogy approach and a modified coupled-field finite element approach (Freed and Babuska 1997). The thermal analogy has been shown to be reasonably accurate in terms of static deformation but transient behaviour can require unwieldy models and accurate modeling of any insulating layers is needed to accurately capture dynamic behaviour Côté et al. (2004). As a result, it has been investigated further and adopted by many different researchers when modeling the piezoelectricinduced strain (Paradies and Ciresa 2009; Tawfik et al. 2011; Bilgen et al. 2011; Gustafson 2011; Dwarakanathan et al. 2015).

Other researchers have looked to improve the modeling accuracy of the MFCs in terms of their electromechanical characteristics (Deraemaeker and Nasser 2010; Prasath and Arockiarajan 2013), their elastodynamics (Steiger and Mokrý 2015; Tan et al. 2018), thermal expansivity (Park and Kim 2007), fatigue properties (Pandey and Arockiarajan 2017), hysteresis and creep (Stuebner et al. 2009; Schrock et al. 2010), nonlinear strain response (Williams et al. 2006; Zheng et al. 2017) and high electric field responses (Nelson et al. 2003). Numerical models and experimental measurements of MFCs have subsequently been investigated on simple unimorph and bimorph structures including bistable 'snap-through' laminates (Schultz and Hyer 2004; Bowen et al. 2006; Giddings et al. 2008; Portela et al. 2008; Senba et al. 2010; Bowen et al. 2011, 2014; Lee et al. 2017) and cantilevers (LaCroix and Ifju 2012; Zhang et al. 2015; Tan et al. 2017). Some modeling works treat the MFC actuation strain as constant, or as a ramped function of the excitation voltage. The actual piezo-strain effect, however, is much more continuous (Wilkie et al. 2004; Williams et al. 2006) and is not symmetric in the negative voltage range Zheng et al. (2017). LaCroix and Ifju (2012, 2015) modeled this continuous response and demonstrated good agreement with FEA and experimental results on unimorph and bimorph structures.

The existing modeling work detailed above has focused predominately on the behaviour of simple beams, cantilevers, and unimorphs. The work on laminates has focused mainly on characterising the snap-through behaviour between bi-stable points. In this paper a constrained encastre 
plate setup is investigated with the aim to develop accurate modeling approaches for continuous, integrated surfaces such as that of a wing. It is important in such a case to ensure minimal disruption to the surface to reduce excrescence drag. To assess the aerodynamic performance of these morphing structures a means of coupling the models with existing fluid dynamics analysis techniques is also desired. To investigate and develop these models a composite laminate skin was manufactured as a test specimen for experimental validation. Whilst the encastre setup is somewhat similar to that of deformable mirrors (Bu et al. 2003; Ma et al. 2011) and fluidic membranes (Xu et al. 2007) the modeling problem is simpler in these cases due to the radial symmetry of the composite.

\section{Paper outline}

The remainder of this paper is composed as follows: in section 2 a description of the test laminate specimen is given. Section 3 then summarises a standard numerical modeling methodology for piezloelectric materials as an analogy to thermal expansion as well as detailing the finite element model using this approach. Section 4 developes the analytical model for this setup using modified bending beam theory, detailing the mothod for modeling the actuation from the MFC patches for both a uniform composite beam of equal lengths of actuator and substrate, and for the specific case investigated here where the lengths are not equal. Section 5 provides experimental validation of the modeling approaches and general discussion on results and the modeling approaches. This includes an approach to estimate the deflection from the experimental strain measurements. In section 6 an application of the analytical model is demonstrated for modeling the aerodynamic performance of a MFC-actuated morphing aerofoil.

\section{Investigative setup}

The encastre plate configuration for this work is illustrated in Figure 3. The laminate 'skin' (parameters associated with which will be denoted with the subscript $s$ ) is an EGlass epoxy resin composite laminate with two plies of 200 gsm $2 / 2$ twill woven glass fiber. This is an advantageous configuration for a morphing wing as it helps ensure a smooth profile between the surface of the wing and laminate skin, eliminating excrescence drag associated with discontinuous surfaces. However, the range of deflection is limited due to the fixture of both ends.

The E-Glass laminate was manufactured using a standard vacuum bagging procedure and then cut to the required

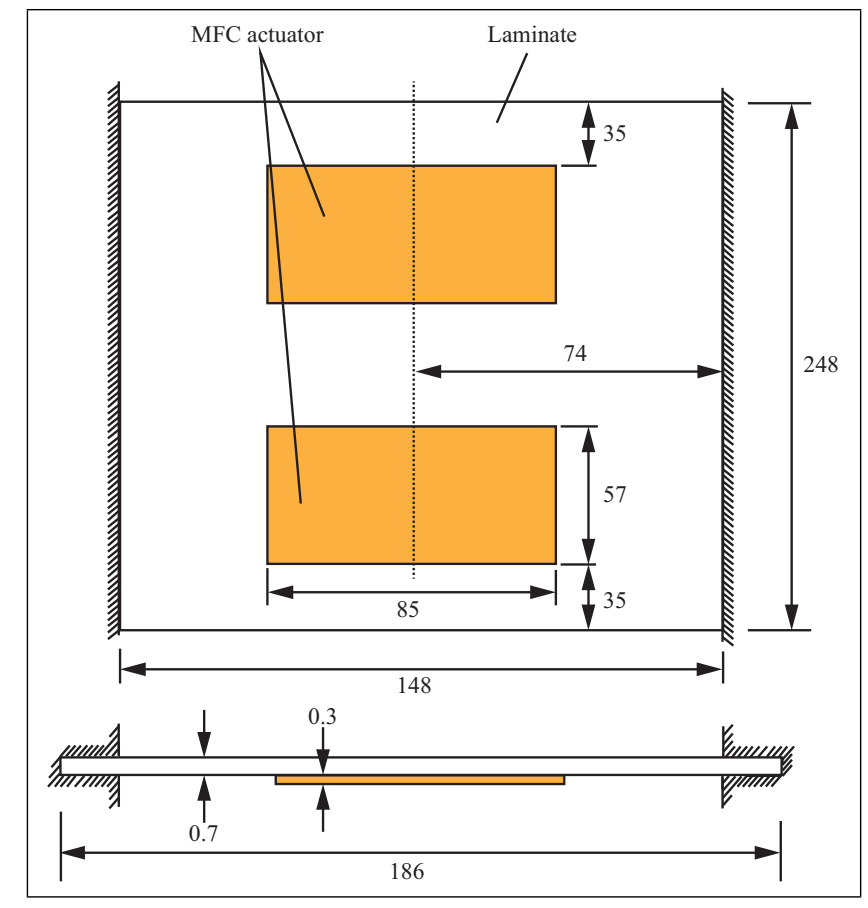

Figure 3. Encastre configuration of the laminate for testing.

Table 1. MFC M8557-P1 specification

\begin{tabular}{|c|c|c|c|}
\hline Active length & & 85 & $\mathrm{~mm}$ \\
\hline Active width & & 57 & $\mathrm{~mm}$ \\
\hline Thickness & & 0.3 & $\mathrm{~mm}$ \\
\hline Free strain* & & 1800 & $\mathrm{ppm}$ \\
\hline Maximum blocking force & & 923 & $\mathrm{~N}$ \\
\hline Voltage range & & -500 to 1500 & V \\
\hline Piezoelectric coefficients & $d_{33}$ & 400 to 460 & $\mathrm{pm} / \mathrm{V}$ \\
\hline
\end{tabular}

${ }^{*}$ For 0 to $1500 \mathrm{~V}$.

dimensions $\left(248 \times 186 \mathrm{~mm}^{2}\right)$. The final thickness after manufacture was measured at $0.7 \mathrm{~mm}$ with an overall mass of $48 \mathrm{~g}$. Tensile testing was performed on a $120 \times 248 \mathrm{~mm}^{2}$ offcut part of the E-glass laminate using a Tinius Olsen 25ST Benchtop Tester (Figure 4a). Three-point bending test results are shown in Figure $4 \mathrm{~b}$ where the gradient of the linear region satisfies the bending beam equation

$$
\frac{\mathrm{d} F}{\mathrm{~d} \delta}=\frac{48 E_{s} I}{L^{3}}
$$

where $L=A B=120 \mathrm{~mm}$ (Figure 4a) and $I=3.43 \times$ $10^{-12} \mathrm{~kg}-\mathrm{m}^{2}$. Solving (1) for $E_{s}$ gives the longitudinal tensile stiffness of the laminate as approximately $12 \mathrm{GPa}$.

Two M8557-P1 type MFC actuators were then adhered to the central region of the E-Glass skin with standard two-part epoxy resin. The M8557-P1 type MFC is polarised for the $d_{33}$ effect for actuation, providing a maximum free strain of about $2000 \mathrm{ppm}$ throughout the voltage range of -500 to $1500 \mathrm{~V}$. Its specifications are listed in Table 1. 


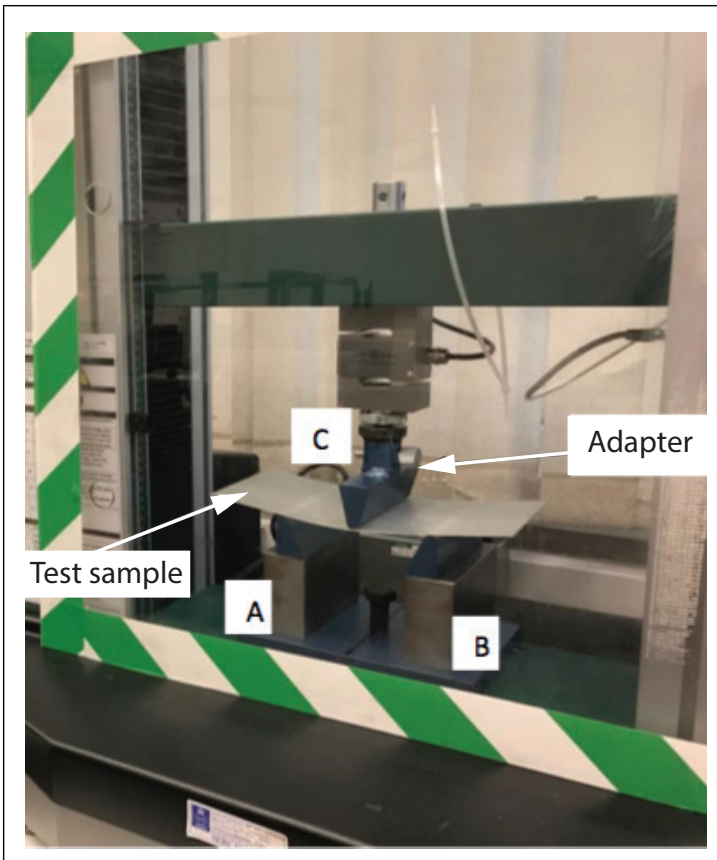

(a) Tinius Olsen 25ST Benchtop Tester.

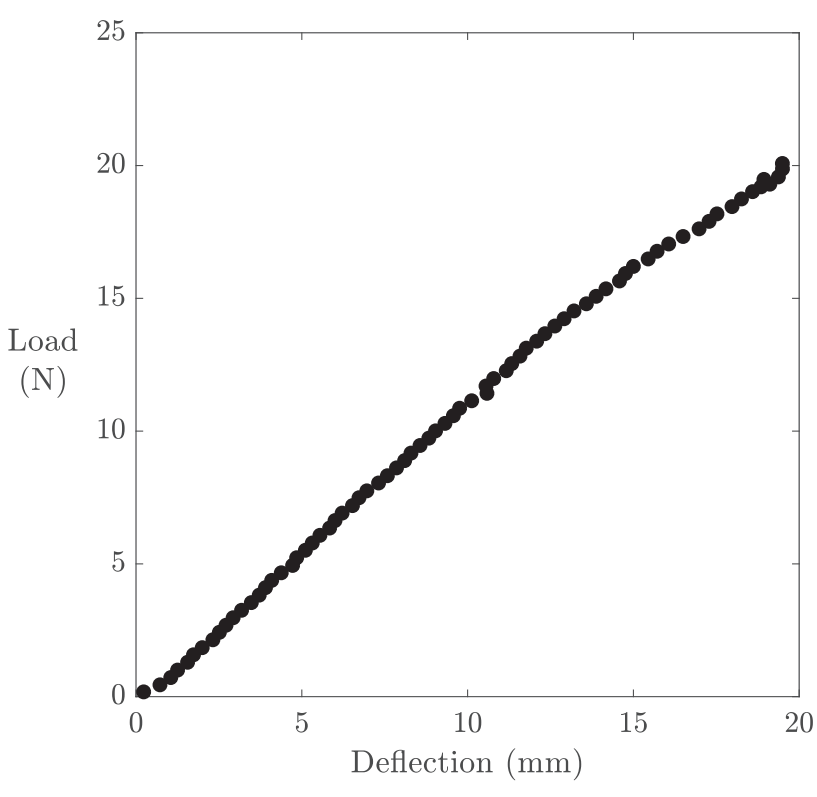

(b) Three-point bending test results.

Figure 4. Measurement of E-glass laminate elastic modulus

\section{Finite element model}

The setup shown in Figure 3 was modeled in the ANSYS Workbench environment. Both the actuator and the EGlass substrate were modeled according to their respective material properties (Table 2). This data was compiled from manufacturing data where possible and then from typical standard values (primarily from Gay and Hoa 2003).

\section{Converse piezoeletric effect by thermal analogy}

Piezoelectric-induced actuation (the converse piezoelectric effect) occurs when an electric input is transformed into mechanical deformation (strain) in an asymmetric crystalline material. This effect can be expressed mathematically by its corresponding constitutive stress equation ANSI/IEEE (1987):

$$
\{T\}=[C]\{S\}-[e]^{\mathrm{T}}\{\mathcal{E}\}
$$

In this equations $\{T\}$ is the $6 \times 1$ stress vector, $[C]$ is the $6 \times 6$ elastic stiffness coefficient matrix in constant electric field condition, $\{S\}$ is the $6 \times 1$ strain vector, $[e]$ is the $3 \times 6$ piezoelectric coupling coefficient matrix, $\{\mathcal{E}\}$ is the $3 \times 1$ electric field vector. Moreover, $[e]^{\mathrm{T}}$ can be written as

$$
[e]^{\mathrm{T}}=[C][d]^{\mathrm{T}}
$$

where $[d]$ is the $3 \times 6$ strain-charge form of the piezoelectric coupling matrix. The converse piezoelectric effect (2) may then be written

$$
\{T\}=[C]\left(\{S\}-[d]^{\mathrm{T}}\{\mathcal{E}\}\right)
$$

Equation 4 is essentially Hooke's law but with consideration of the piezoelectric strain also. It closely resembles the form of Hooke's law with consideration of temperature-induced strain, which has the form

$$
\{T\}=[C](\{S\}-\{\alpha\} \Delta \theta)
$$

where $\{\alpha\}$ is the $6 \times 1$ coefficient of thermal expansion vector and $\Delta \theta$ is the variation in temperature from a reference. Comparing (4) and (5), a similarity between piezoelectric and thermal strains is accomplished:

$$
[d]^{\mathrm{T}}\{\mathcal{E}\}=\{\alpha\} \Delta \theta
$$

Typically, piezoelectric materials such as MFC actuators are polarised in direction 3 (Figure 2), showing a symmetric hexagonal crystallographic structure. As a consequence of this symmetry, there is a significant decrease in the number of independent coefficients in many of the matrices, and (6) 
Table 2. Model laminate and MFC material properties.

\begin{tabular}{lcccc}
\hline Property & & E-Glass-epoxy ply & Epoxy resin ply & M8857-P1 \\
\hline Thickness $(\mathrm{mm})$ & $t$ & 0.2 & 0.15 & 0.3 \\
Density $\left(\mathrm{g} / \mathrm{cm}^{3}\right)$ & $\rho$ & 1.5 & 1.16 & 5.44 \\
Young's modulus $(\mathrm{GPa})$ & $E_{x}$ & 18 & 3.8 & 30.336 \\
& $E_{y}$ & 18 & 3.8 & 15.857 \\
& $E_{z}$ & 10 & 3.8 & 9.514 \\
Poisson's ratio (-) & $\nu_{x y}$ & 0.21 & 0.35 & 0.31 \\
& $\nu_{y x}$ & 0.21 & 0.35 & 0.16 \\
& $\nu_{x z}$ & 0.21 & 0.35 & 0.31 \\
& $\nu_{z x}$ & 0.21 & 0.35 & 0.16 \\
& $\nu_{y z}$ & 0.4 & 0.35 & 0.31 \\
& $\nu_{z y}$ & 0.4 & 0.35 & 0.16 \\
Shear Modulus (GPa) & $G_{x y}$ & 2.22 & 1.4 & 5.515 \\
& $G_{y z}$ & 2.22 & 1.4 & 2.75 \\
& $G_{x z}$ & 2.22 & 1.4 & 2.75 \\
\hline
\end{tabular}

becomes

$$
\left[\begin{array}{ccc}
0 & 0 & d_{31} \\
0 & 0 & d_{32} \\
0 & 0 & d_{33} \\
0 & d_{24} & 0 \\
d_{15} & 0 & 0 \\
0 & 0 & 0
\end{array}\right]\left\{\begin{array}{c}
\frac{\Delta V_{1}}{x_{1}} \\
\frac{\Delta V_{2}}{x_{2}} \\
\frac{\Delta V_{3}}{x_{3}}
\end{array}\right\}=\left\{\begin{array}{c}
\alpha_{1} \\
\alpha_{2} \\
\alpha_{3} \\
\alpha_{4} \\
\alpha_{5} \\
\alpha_{6}
\end{array}\right\} \Delta \theta
$$

The parameter $\mathcal{E}_{i}=\Delta V_{i} / x_{i}$ is the electric field, comprising the voltage difference, $\Delta V_{i}$, and the electrode separation, $x_{i}$, in each axis $i$. In reality, for cases in which the actuator works principally in one direction, it is polarised in direction 3 and the in-plane electric fields $\mathcal{E}_{1}$ and and $\mathcal{E}_{2}$ are assumed to be zero. For this reason, and particularly for this work with a P1 type MFC actuator, the final equality between thermal and piezoelectric strain can be represented mathematically as

$$
d_{3 i} \frac{\Delta V}{x_{e}}=\alpha_{i} \Delta \theta
$$

where $x_{e}=x_{3}$, the electrode separation along direction 3 . There is a variety of different values quoted in the literature for the electrode separation of MFCs, $x_{e}$, owing to size and configuration differences in actuator types. For example, Bilgen (2010) measured a value of $0.46 \mathrm{~mm}$, whilst Williams (2004) states a value of $0.533 \mathrm{~mm}$.

The relationship of (7) concludes that the piezoelectricdriven expansion and contraction can be represented through a thermal load applied to the modeled actuator (Côté et al. 2004). This is quite useful when working with commercial FE software since it allows the piezoelectric actuation to be treated as a thermal expansion property of the material. An applied voltage is input as a thermal load and piezoelectric strain coefficient characterising an actuator is modeled as thermal expansion coefficient determined by the relationship

$$
\alpha_{i}=\frac{S_{i_{\max }}}{\Delta \theta_{\max }} \equiv \frac{S_{i_{\max }}}{\Delta V_{\max }}=\frac{d_{3 i}}{x_{e}}
$$

for $i=1,2,3$. Let oxyz define the engineering axes along which strain is considered, such that, for the P1 type actuator, $o x \equiv o 3, o y \equiv o 2$, and $o z \equiv o 1$ (see Figure 2). Then, $\Delta V=$ $\Delta V_{3}$ and

$$
\begin{gathered}
S_{x}=d_{33} \frac{\Delta V}{x_{e}} \equiv \alpha_{x} \Delta V, \quad S_{y}=d_{32} \frac{\Delta V}{x_{e}} \equiv \alpha_{y} \Delta V, \\
\text { and } \quad S_{z}=d_{31} \frac{\Delta V}{x_{e}} \equiv \alpha_{z} \Delta V
\end{gathered}
$$

The piezoelectric coefficients are not constant and exhibit a nonlinearity with respect to the excitation voltage. All MFCs have a rated value of $400 \mathrm{pm} / \mathrm{V}$ for the longitudinal piezoelectric coefficient $\left(d_{33}\right)$, rising to $460 \mathrm{pm} / \mathrm{V}$ when exposed to a DC electric field above $1 \mathrm{kV} / \mathrm{mm}$ (i.e., $\mathcal{E}_{3} x_{e} \approx$ $500 \mathrm{~V}$ for the P1 type). These piezoelectric constants correspond roughly to the specified free strain per volt values of $0.75 \mathrm{ppm} / \mathrm{V}$ and $0.9 \mathrm{ppm} / \mathrm{V}$. For P1 type MFCs orthogonal strain also occurs due to the $d_{31}$ and $d_{32}$ coefficients. Rated values for the transverse coefficient $\left(d_{31}\right)$ are similarly -170 $\mathrm{pm} / \mathrm{V}$ and $-210 \mathrm{pm} / \mathrm{V}$, with equivalent strain per volt values of approximately $-0.32 \mathrm{ppm} / \mathrm{V}$ and $-0.4 \mathrm{ppm} / \mathrm{V}$. To a reasonably good approximation, it is found for all MFC types that $d_{31} / d_{33} \approx-0.45$ (Bowen et al. 2011). Also, $d_{31} \approx d_{32}$. However, these should be taken as approximate values, useful for simple modeling in the mid-operating voltage range, as the coefficients vary in a continuous fashion with the electric field (Figure 5). There is some variation in the reported values of the piezoelectric coefficients in the literature which could be due to a myriad of reasons. Since the piezoelectric coefficients are heavily influenced by 


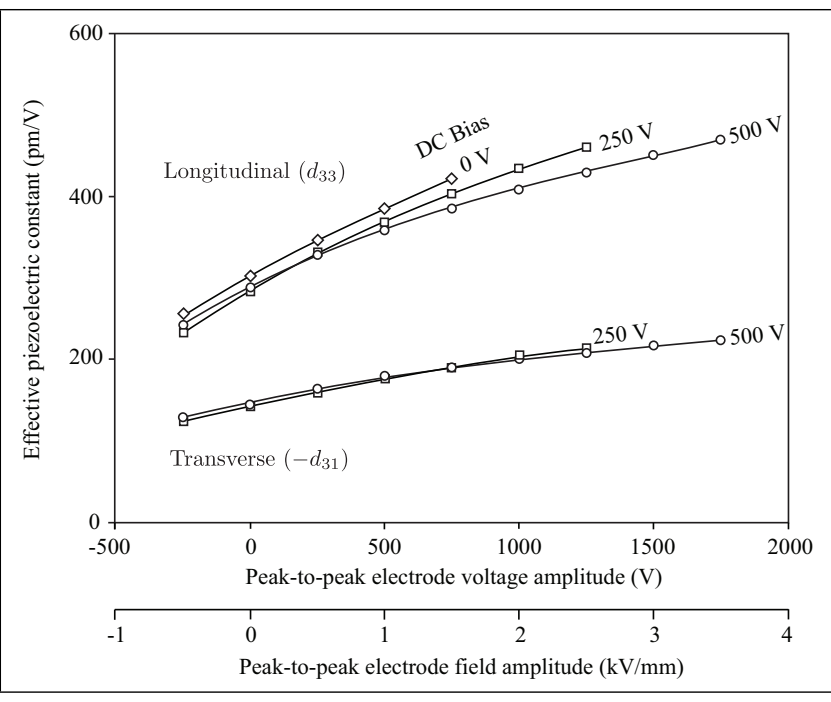

Figure 5. Variation of $d_{33}$ and $d_{31}$ piezoelectric coefficient with peak-to-peak voltage across the electrodes and DC bias. Data compiled from Williams (2004) and Wilkie et al. (2004).

voltage, excitation cycling, and mechanical loading, various nonlinearities, and by definition the electrode spacing, it should not be surprising there is moderate variation in the values reported in the literature. Also, the researcher should take care in how the coefficients are defined in each case; in particular, whether they are expressed as effective or normalised (secant) coefficients. For example, the values presented in LaCroix and Ifju (2015) are in normalised form. The variation in the reported values of $x_{e}$ will also have a noticeable effect on the stated values if they are derived from strain data. Therefore, when working with a specific device and in the absence of an accurate measurement of $x_{e}$, it is more convenient to work with the strain per volt characteristic, $\alpha$, which should be consistent across all MFC devices. This is also more pertinent to FEA modeling using the thermal analogy.

\section{MFC model}

The MFC was modeled as an orthotropic uniform material. The mechanical properties of MFCs have been investigated in several other works, all reporting some minor variation. A summary of these is given by Steiger and Mokrý (2015) whose own FEM modeling gives the most complete set of mechanical characteristics for the MFC to date. In this work the manufacture's specified mechanical properties are used but with the following assumptions: $E_{z}=0.6 E_{y}, \nu_{y z}=$ $\nu_{x z}=\nu_{x y}, \quad \nu_{z y}=\nu_{z x}=\nu_{y x}$, and $G_{y z}=G_{x z}=G_{x y} / 2$. These assumptions are made based on the results presented by Steiger and Mokrý (2015).

The subscript $p$ will be used to denote parameters associated with the piezoelectric MFC. Using the manufacturer's rated specification (namely, Table 1), and the mechanical and piezoelectric properties also stated, the rated performance (which corresponds to peak-to-peak operation),

$$
\begin{gathered}
S_{x_{\max }}=\int_{0}^{1500} \frac{d_{33}}{x_{e}} \mathrm{~d} V=1800 \mathrm{ppm}, \\
F_{b}=S_{x_{\max }} A_{p_{x}} E_{p_{x}}=923 \mathrm{~N} \\
\text { and } \Delta L_{x_{\max }}=S_{x_{\max }} L_{p} \approx 150 \mu \mathrm{m},
\end{gathered}
$$

can be observed when $t_{p}=0.2965 \mathrm{~mm}$ and $x_{e}=0.367 \mathrm{~mm}$. Whilst the thickness agrees well with the typical values of $t_{p}$ of $0.3 \mathrm{~mm}$, the electrode separation value appears quite smaller than typically quoted values. The strain response from this linear-ramp model is shown in Figure 6 as the broken trace. It can be seen that the size of $x_{e}$ has a significant effect on the strain response and likely explains the variations in $d_{33}$ values reported in the literature.

The nonlinearity in the $d_{33}$ (and $d_{31}$ ) effect was initially based on the reported behaviour by the works by Williams et al. (Williams 2004; Wilkie et al. 2004; Williams et al. 2006). However, the values reported in these works are commonly referred to as 'effective' coefficients, obtained by examining cyclic peak-to-peak strain response of varying voltage ranges about a DC bias. This form is of particular use when quantifying the performance of such piezoelectric systems as sensors (Nguyen et al. 2017). Equation (4) comprises the coefficients in 'normalised' form, obtained by directly examining the strain per volt relationship. However, we note that by scaling the effective coefficient values the desired specification performance can be achieved. This was found with values of $d_{33}$ at a factor of $2582 x_{e}$ greater

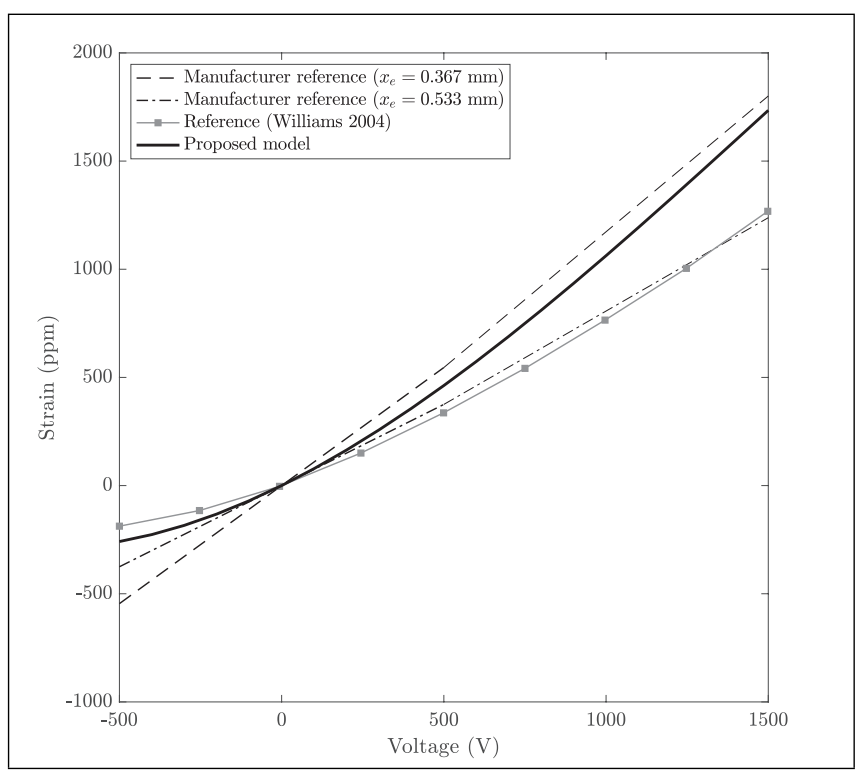

Figure 6. Modeled longitudinal $\left(d_{33}\right)$ strain response with voltage across the electrodes. 
than the effective coefficient values reported by Williams (2004). A similar procedure was also found performed by Latalski (2011) in order to achieve modeling parity for an M8503-P1 MFC by scaling the manufacturer specification. Importantly, the nonlinear effect at both lower and higher voltages is adequately captured. This is evident in the proposed model in Figure 6 which follows the strain performance suggested by the manufacturer's specification (with $x_{e}=0.367 \mathrm{~mm}$ ) more closely than the initial model based on data from Williams (2004); Wilkie et al. (2004). However, those coefficients (with values as shown in Figure 5) match the manufacturer specifications when $x_{e}=0.533$ $\mathrm{mm}$, illustrating the significance the size of $x_{e}$ has on the performance and modeling accuracy.

It can be seen that for negative voltages capturing the nonlinear behaviour in the strain model is important as the curve slopes start to noticeably deviate from the linear step model. Given these values the equivalent values of $\alpha_{x}$ are given in Table 3 . These values compare reasonably well with the values obtained by LaCroix and Ifju (2015). However, based on their experiments they used the average strain response between hysteresis curves in their simulations, whereas this data set is more suited to the actual strain response on the upgoing hysteresis curve. Consequently, their values have larger magnitude in the lower voltage range. Their data also exhibits a non-monotonic change in the slope which is typical of most piezoelectric materials. However, this behaviour is not always observed in other works detailing the use of MFC, (e.g. Williams et al. 2006). Inspecting $d_{31} / d_{33}$ from the data points in Figure 5 gives a mean ratio of -0.49 , which is reasonably close to the oft-stated value of -0.45 . Hence, $\alpha_{y}=\alpha_{z}=-0.49 \alpha_{x}$. The proposed polynomial model for the strain per volt response in each axis is

$$
\begin{aligned}
& \alpha_{x}(V) \\
& \quad=\left(-0.0881 V^{2}+407.56 V+743180\right) \times 10^{-12} \\
& \begin{aligned}
\alpha_{y}(V) & =\alpha_{z}(V) \\
& =\left(0.0432 V^{2}-199.7 V-364160\right) \times 10^{-12}
\end{aligned}
\end{aligned}
$$

These values for $\alpha$ were then used as secant coefficient of thermal expansion property for the MFC material with a reference temperature of $0 \mathrm{~K}$ (Table 3 ). In this way a temperature input may be used to represent voltage inputs, as per (8). Note that these values of $\alpha$ are only valid when the zero-strain voltage is zero (hence, the $\Delta$ prefix on $V$ has been dropped). Therefore, the model assumes zero-strain at
Table 3. Secant coefficient of thermal expansion values for MFC FE model.

\begin{tabular}{rcc}
\hline$\Delta \theta(\mathrm{K})$ & $\alpha_{x}\left(\times 10^{-6} \mathrm{~K}^{-1}\right)$ & $\alpha_{y}=\alpha_{z}\left(\times 10^{-6} \mathrm{~K}^{-1}\right)$ \\
\hline-500 & 0.517 & -0.253 \\
-400 & 0.566 & -0.277 \\
-300 & 0.613 & -0.300 \\
-200 & 0.658 & -0.322 \\
-100 & 0.701 & -0.344 \\
0 & 0.743 & -0.364 \\
100 & 0.783 & -0.383 \\
200 & 0.821 & -0.402 \\
300 & 0.857 & -0.420 \\
400 & 0.892 & -0.437 \\
500 & 0.925 & -0.453 \\
600 & 0.956 & -0.468 \\
700 & 0.985 & -0.483 \\
800 & 1.013 & -0.496 \\
900 & 1.038 & -0.509 \\
1000 & 1.062 & -0.521 \\
1100 & 1.085 & -0.532 \\
1200 & 1.105 & -0.542 \\
1300 & 1.124 & -0.551 \\
1400 & 1.141 & -0.559 \\
1500 & 1.156 & -0.567 \\
\hline
\end{tabular}

$0 \mathrm{~V}$ and any data used for comparison should be adjusted accordingly. The model is also only valid for increasing voltages corresponding to upgoing hysteresis curve. A more comprehensive model incorporating hysteresis effects is not readily useable with the thermal analogy in most FE software and would require something akin to the Preisach model of hysteresis (Zheng et al. 2017).

\section{Laminate model}

The composite skin was modeled using ANSYS composite Prep/Post (ACP). The thickness of E-glass weave, per woven ply, was defined as $0.2 \mathrm{~mm}$ according to the manufacturer data [29]. Since the overall thickness of the final E-glass laminate was $0.7 \mathrm{~mm}$, it is assumed the two E-glass plies are effectively sandwiched between two $0.15 \mathrm{~mm}$ epoxy layers, representing the excess resin. The proposed stacking arrangement is illustrated in Figure 7. Material properties for the epoxy resin were based on the default ANSYS values which were similar to the manufacturers specification (Table 2).

The total mass of the laminate ( $48 \mathrm{~g}$ ) along with its volume $\left(248 \times 186 \times 0.7 \mathrm{~mm}^{3}\right)$ gives a density of $1.49 \mathrm{~g} / \mathrm{cm}^{3}$, which is lower than typical manufactured fiber-glass laminates $(\sim 2$ $\left.\mathrm{g} / \mathrm{cm}^{3}\right)$. This can be explained by the excess resin in the laminate. Each resin ply is believed to be isotropic, 0.15 $\mathrm{mm}$ thick, have a density, $\rho_{m}$, of $1.15 \mathrm{~g} / \mathrm{cm}^{3}$ and a Young's modulus, $E_{m}$, of $3.8 \mathrm{GPa}$. Each resin ply has a volume of 


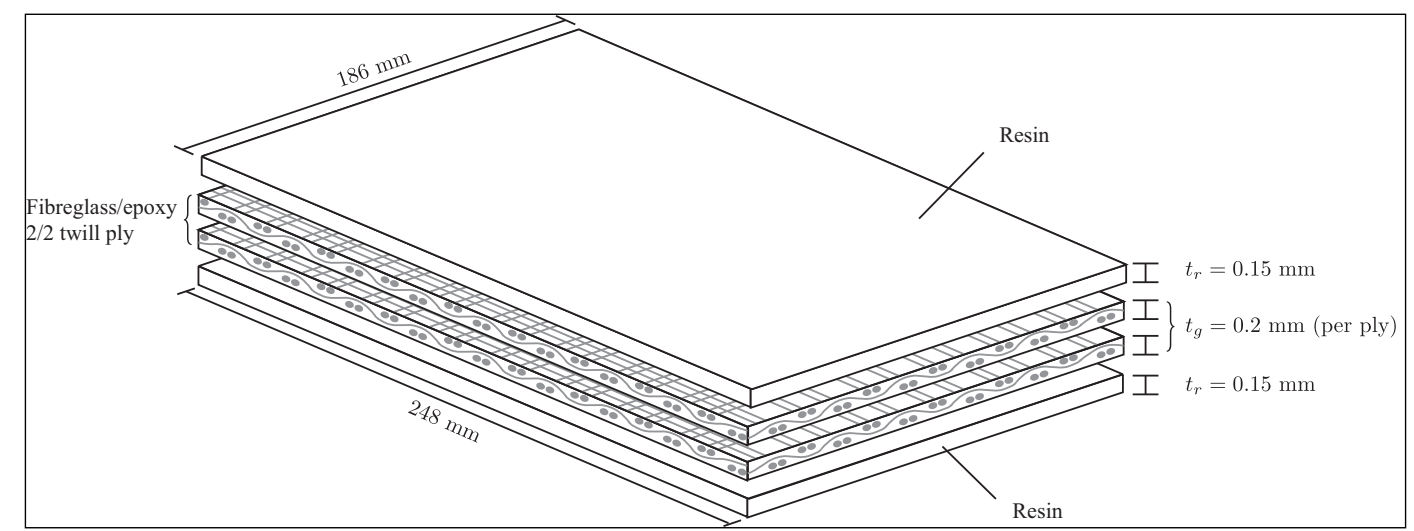

Figure 7. Exploded view of plies in the modeled laminate.

$6919 \mathrm{~mm}^{3}$ and, hence, a mass of $7.95 \mathrm{~g}$ (assuming no voids). The estimated mass of each fiberglass ply is then $\sim 16 \mathrm{~g}$. The mass of the E-glass weave in each E-glass/epoxy ply is estimated as $9.25 \mathrm{~g}$ (based on manufacturer data) giving a mass fraction and volume fraction ratio for the fiber content of the laminate as $M_{f}=0.58$ and $V_{f}=0.39$. Resin matrix ratios for the E-glass plies are then $M_{m}=1-M_{f}=0.42$ and $V_{m}=1-V_{f}=0.61$. Using the rule of mixtures gives the elastic moduli characteristics per unidirectional lamina as follows:

$$
\begin{aligned}
E_{x_{u}} & =E_{f} V_{f}+E_{m} V_{m}=30.04 \mathrm{GPa}, \\
E_{y_{u}} & =\frac{Y_{m}}{\left(1-V_{f}\right)+\frac{Y_{m}}{E_{f}} V_{f}}=5.98 \mathrm{GPa}, \\
G_{x y_{u}} & =\frac{G_{m}}{\left(1-V_{f}\right)+\frac{G_{m}}{G_{f}} V_{f}}=2.21 \mathrm{GPa},
\end{aligned}
$$

where $E_{f}=72 \mathrm{GPa}$ and $G_{f}=30 \mathrm{GPa}$ are typical values for glass fiber and $E_{m}=3.8 \mathrm{GPa}$ and $G_{m}=1.4 \mathrm{GPa}$ are typical values for the epoxy resin matrix. Similarly the poisson ratio is found by taking $\nu_{f}=0.25$ and $\nu_{m}=0.35$ :

$$
\nu_{x y_{u}}=\nu_{f} V_{f}+\nu_{m} V_{m}=0.31
$$

The properties of the E-glass ply are then estimated by compositing two orthogonal unidirectional fabrics into one weave. If $n_{1}$ is the number of warp yarns per metre and $n_{2}$ is the number of weft yarns per metre, for a balanced $2 / 2$ twill weave, $k=n_{1} /\left(n_{1}+n_{2}\right)=0.5$ and the elastic properties are isotropic:

$$
\begin{gathered}
E_{x_{g}}=E_{y_{g}}=k E_{x_{u}}+(1-k) E_{y_{u}}=18.01 \mathrm{GPa}, \\
G_{g}=G_{x y_{u}},
\end{gathered}
$$

and

$$
\nu_{g}=\frac{\nu_{x y_{u}}}{k+(1-k) \frac{Y_{x_{u}}}{Y_{y_{u}}}}=0.103 .
$$

The resulting properties of the laminate, taking into account the excess resin plies, are then similarly obtained by the rule of mixtures:

$$
E_{s}=E_{x_{g}} \frac{t_{g}}{t}+E_{m} \frac{t_{r}}{t}=11.9 \mathrm{GPa}
$$

where $t, t_{g}$, and $t_{r}$ are the total thickness of the laminate and the thickness of the E-glass and resin plies, respectively. Also, $G_{s}=G_{x y}$ and

$$
\nu_{s}=\nu_{g} \frac{t_{g}}{t}+\nu_{m} \frac{t_{r}}{t}=0.21 .
$$

Note that an almost identical value for $E_{s}$ may be obtained by considering the laminate as two glass fiber/epoxy composite plies of thickness $0.35 \mathrm{~mm}$ with $M_{f}=0.38$ and $V_{f}=0.22$. This value of $E_{s}$ agrees very well with the experimental measurement determined by the three point bending test. Regardless, since the stiffness is known not to significantly determine the deflection characteristics, a small uncertainty is acceptable. A summary of the material modeling properties are given in Table 2.

Once the material properties and geometry were defined the next step was to create the laminate in ANSYS ACP (Figure 8a). This was then exported to a 'static structural' model with solid composite elements for analysis in the ANSYS Workbench environment. A standard quadrilateral mesh was used for both laminate and MFC. Mesh size for both were set to $1 \mathrm{~mm}$ which provided reasonable solution times and minimal improvement in solutions below this mesh size. The end faces of the laminate where then constrained and a thermal load of varying temperature applied to both actuator materials (Figure $8 b$ ). 


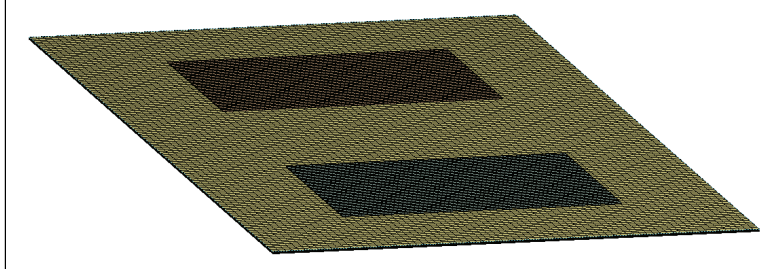

(a) Mesh.

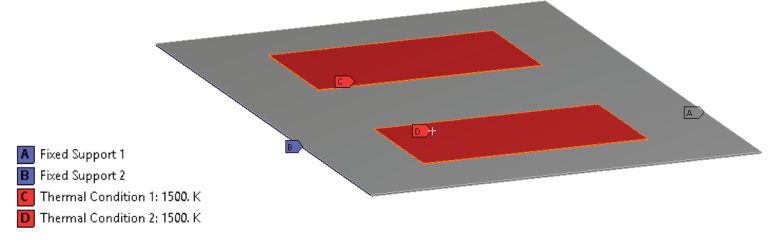

(b) Boundary constraints.

Figure 8. Finite element model construction in ANSYS.

Figure 9 shows the deflection and strain response to the excitation range across the longitudinal centreline through the laminate. The nonlinear characteristics at the negative polarity are represented, with the deflection and strain being approximately half that at $500 \mathrm{~V}$. Although the actual change in the magnitude of the deflection between $1000 \mathrm{~V}$ to 1500 $\mathrm{V}$ is only $63 \%$, compared to $130 \%$ from $500 \mathrm{~V}$ to 1000 $\mathrm{V}$, the relative change (i.e. gradient) is slightly larger (2.5 $\mathrm{mm} / \mathrm{kV}$ compared to $2.24 \mathrm{~mm} / \mathrm{kV}$ ). This behaviour follows the variation of $\alpha_{x}$ since the relationship with the deflection is entirely linear.

The transverse deflections evident in Figure 10 and Figure 11 are due to both transverse curvature and the $d_{31}$. These are, however, generally small. The contraction that occurs in the transverse axis at positive voltages results in some minor transverse compression on the laminate surface, This causes minor deformation on the transverse sides of the MFCs. Consequently, there is some variation in longitudinal deformation across the laminate's span (approximately 0.5 $\mathrm{mm}$ ). Between the centreline and edges of the laminate there is minor difference and, since both longitudinal ends of the laminate are constrained, this difference is reasonably small. However, the deflection in both central and end planes is noticeably larger than the deformation in the quarter-line planes that run through the MFCs.

Results were also compared to a solid laminate model and were in very good agreement with the ACP model, suggesting the stacking order in Figure 7 is a reasonable representation of the actual laminate composition. Also, since the deflections are small, the inter-ply interactions are not of particular interest in this study. The accuracy of the ply stacking model is therefore not crucial. A comparison with experimental results is given after the next section.

\section{Analytical model}

An analytical approach to modeling the plate deflection is desirable for quick, preliminary analysis of surface deformation. This is particularly useful when the structure interacts with fluid and two-dimensional coupling of the structural and fluid dynamics models is required (an example is illustrated later in $\S$ ). As such, a comprehensive model of the three dimensional plate deformation is beyond the scope of this investigation. Instead, a simple one-dimensional bending model based on beam statics is sufficient. Prior to this, the actuation on the beam model must be determined.

\section{Free composite plate bending model (equal actuator and substrate lengths)}

Consider a composite plate comprising a MFC actuator and a substrate, both of equal plane dimensions as illustrated in Figure 12. Along the $x$ axis a force $P_{p_{x}}$ is created by the straining actuator after the electric field is applied, producing a resultant force $P_{s_{x}}$ at the ends of the substrate. The resulting couple defines the moment $M_{x}$ acting at both ends of the plate. The equivalent is true in the $y$ axis. The thickness of the actuator and substrate are denoted $t_{p}$ and $t_{s}$, respectively, and $w_{p}$ is the width of the cross section, which is the same for both materials in this case. However, note that when considering the bending in the $y$ direction, the width is $L_{p}$.

It is conventional in beam and plate analysis to often treat moments causing upwards curvature as negative. This results from the $z$ axis being orientated downwards. Due to the axes definitions shown in Figure 12, where $M_{x}$ refers to the moment in the $x$ direction, positive moments are taken clockwise about the normal to the plane of motion (i.e. about the $y$ axis). Then, beams that hog will have negative curvature. Distances above the neutral axis are taken to be positive so that strains above the neutral axis are correspondingly positive (tensile). The bending strain in the plate is then generally given as $-\kappa_{(\cdot)}\left(z-z_{n_{(\cdot)}}\right)$ where $\kappa_{(\cdot)}$ and $z_{n_{(\cdot)}}$ are the curvature and neutral axis in the plane $(\cdot) z$.

For orthotropic plates that experience direct bending only (i.e. no twisting) the moment-curvature equations per unit width are

$$
\{M\}=[D]\{\kappa\}
$$




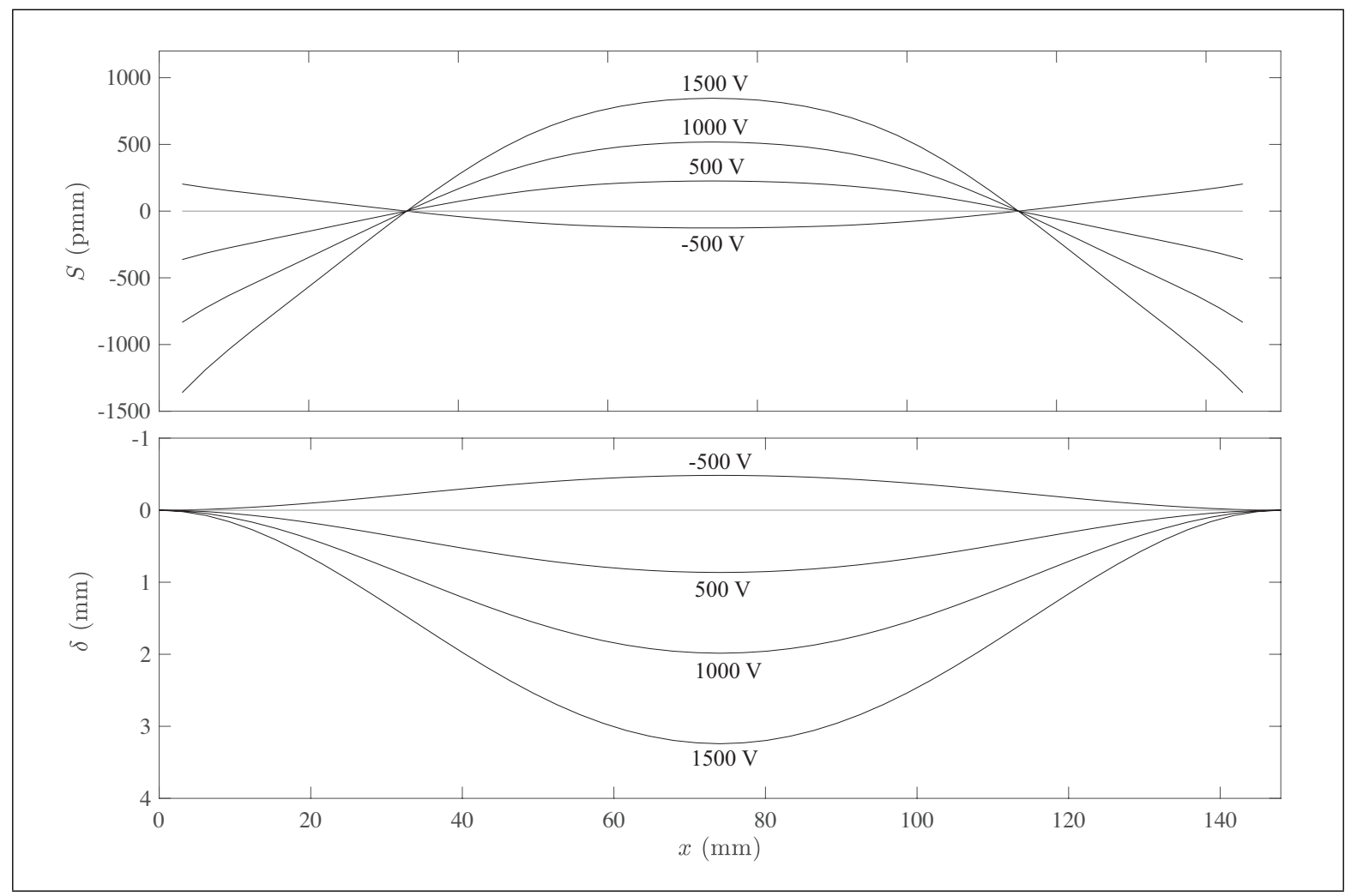

Figure 9. Deflection and strain results from FE model.

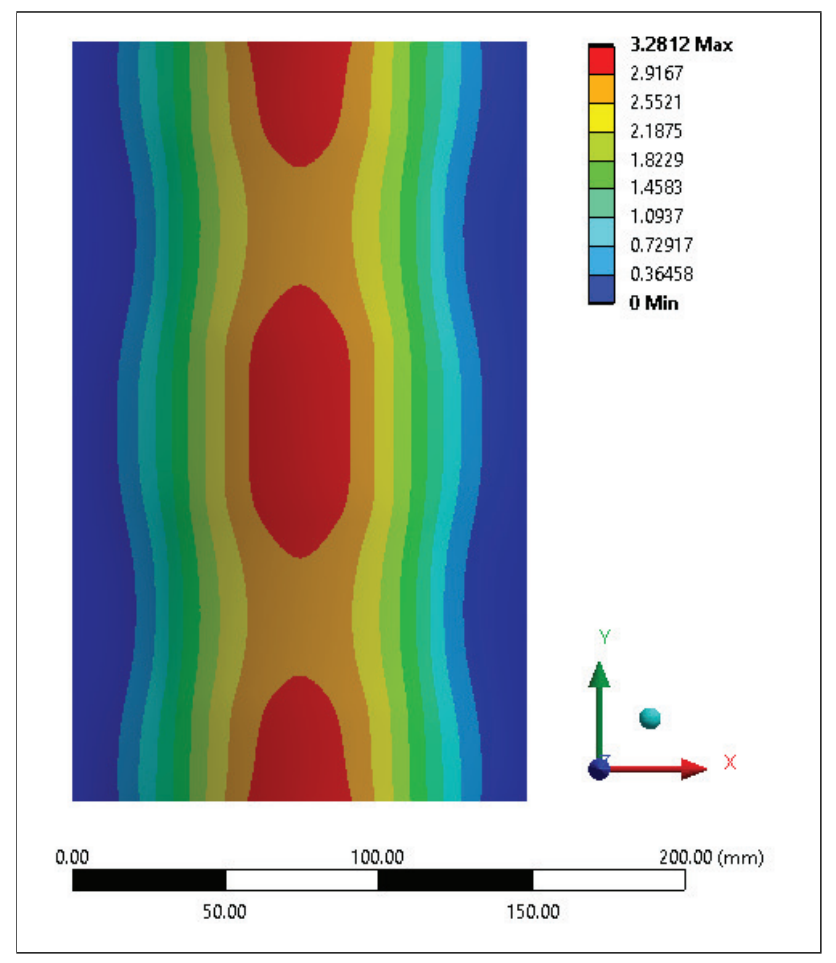

Figure 10. Simulated surface deflection on the laminate at $1500 \mathrm{~V}$.
$D_{(\cdot)}$ are the plate flexural rigidity coefficients per unit width. Specifically,

$$
[D]=\frac{t^{3}}{12\left(1-\nu_{x y} \nu_{y x}\right)}\left[\begin{array}{cc}
E_{x} & \nu_{y x} E_{x} \\
\nu_{x y} E_{y} & E_{y}
\end{array}\right]=\frac{t^{3}}{12}[C]
$$

where $\nu_{(\cdot)}$ are the Poisson's ratios of the strains in the $x$ and $y$ axes. Given that

$$
\kappa_{x}=\frac{1}{r_{x}}=\frac{\partial^{2} \delta}{\partial x^{2}} \quad \text { and } \quad \kappa_{y}=\frac{1}{r_{y}}=\frac{\partial^{2} \delta}{\partial y^{2}}
$$

then

$$
\left\{\begin{array}{c}
\frac{\partial^{2}}{\partial x^{2}} \\
\frac{\partial^{2}}{\partial y^{2}}
\end{array}\right\} \delta=\frac{12}{t^{3}}[C]^{-1}\{M\}
$$

where $[C]^{-1}$ is the compliance matrix. This expands to

$$
\begin{aligned}
\frac{\partial^{2} \delta}{\partial x^{2}} & =\frac{12}{t^{3}} \frac{M_{x}}{E_{x}}-\frac{12}{t^{3}} \frac{\nu_{x y} M_{y}}{E_{x}} \\
& =\frac{w_{p}}{E_{x} I_{x}}\left(M_{x}-\nu_{x y} M_{y}\right)=\frac{M_{x}-\nu_{x y} M_{y}}{D_{x}\left(1-\nu_{x y} \nu_{y x}\right)} \\
\frac{\partial^{2} \delta}{\partial y^{2}} & =-\frac{12}{t^{3}} \frac{\nu_{y x} M_{x}}{E_{y}}+\frac{12}{t^{3}} \frac{M_{y}}{E_{y}} \\
& =\frac{L_{p}}{E_{y} I_{y}}\left(M_{y}-\nu_{y x} M_{x}\right)=\frac{M_{y}-\nu_{y x} M_{x}}{D_{y}\left(1-\nu_{x y} \nu_{y x}\right)}
\end{aligned}
$$




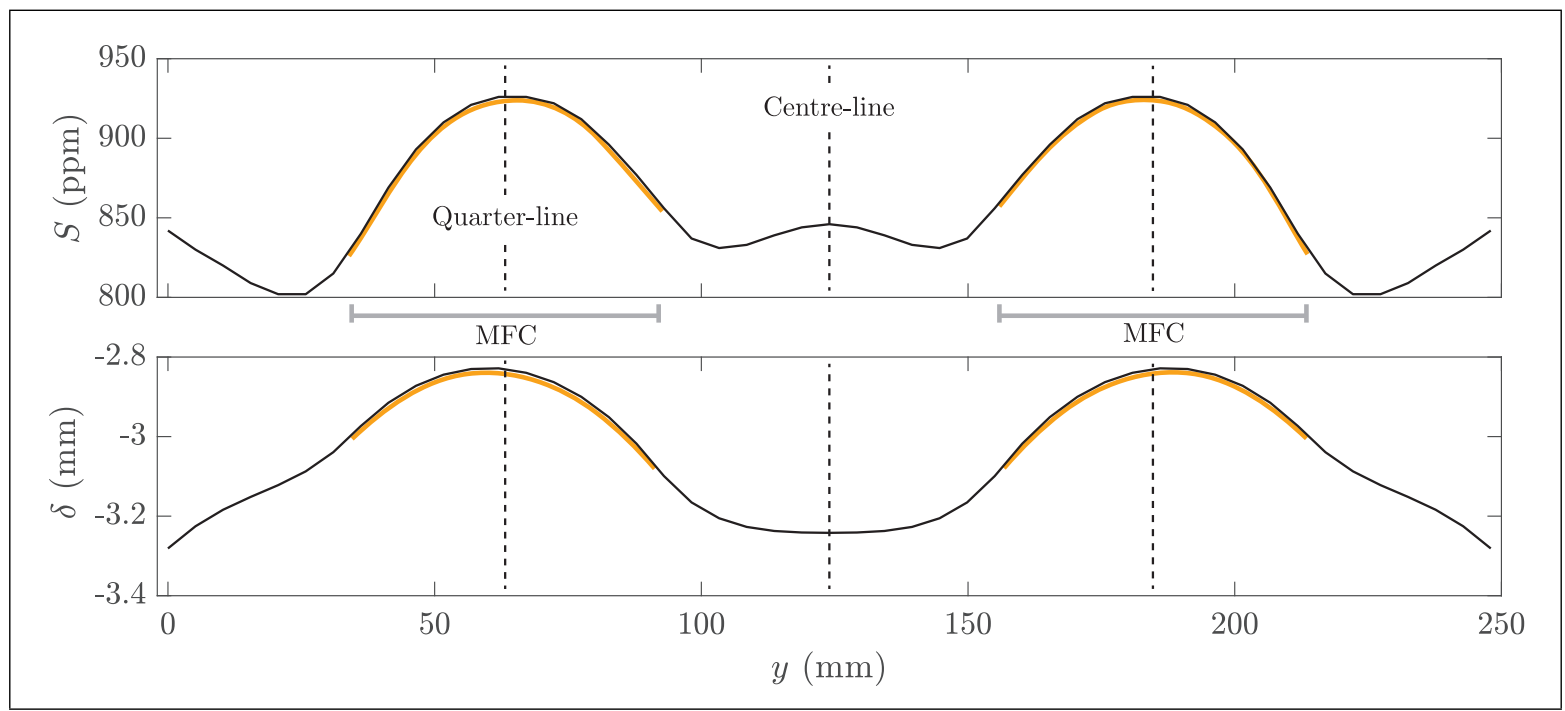

Figure 11. Variation in strain and deflection along the transverse centre-line from FE model for $1500 \mathrm{~V}$ excitation. Locations of the MFC actuators are shown.

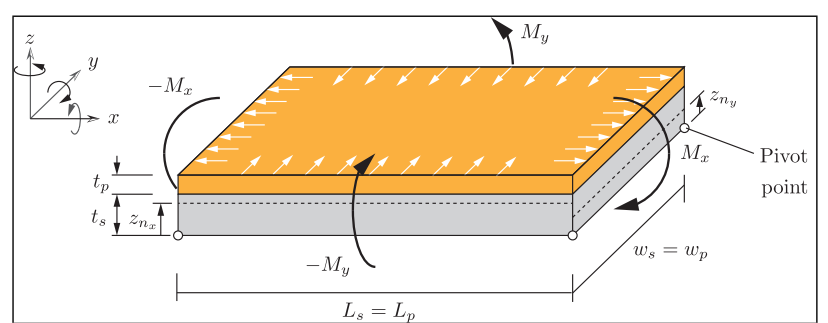

Figure 12. Diagram of the composite actuator free bending model.

which may be rearranged to determine the moments of interest:

$$
\begin{aligned}
M_{x}=\kappa_{x} D_{x}\left(1-\nu_{x y} \nu_{y x}\right)+ & \nu_{x y} M_{y} \\
& =\kappa_{x} D_{x}+\nu_{x y} \kappa_{y} D_{y}, \\
M_{y}=\kappa_{y} D_{y}\left(1-\nu_{x y} \nu_{y x}\right)+ & \nu_{y x} M_{x} \\
& =\kappa_{y} D_{y}+\nu_{y x} \kappa_{x} D_{x} .
\end{aligned}
$$

It now remains to determine the curvatures in each plane, $\kappa_{x}$ and $\kappa_{y}$. The derivation of these follow similarly the work by DeVoe and Pisano (1997); Weinberg (1999).

Consider the moments of an equivalent beam acting in the $x z$ plane. In Figure 12 it is assumed the bonding between the MFC actuator and laminate is perfect and, hence, negligible. The properties of the epoxy resin used to bond the surfaces are hence not considered in the analysis. Since the curvature of both actuator and substrate is the same, the curvature of a beam caused by the piezoelectric strain may be written

$$
\kappa_{x}=\frac{M_{p_{x}}}{E_{p_{x}} I_{p_{x}}}=\frac{M_{s_{x}}}{E_{s_{x}} I_{s_{x}}} .
$$

The combined flexural stiffness of the composite beam, $E_{x} I_{x}$, can be found by noting

$$
E_{x} I_{x}=E_{s_{x}} I_{s_{x}}+E_{p_{x}} I_{p_{x}}=\frac{w_{s_{x}}}{12}\left(E_{s_{x}} t_{s_{x}}^{3}+E_{p_{x}} t_{p_{x}}^{3}\right) .
$$

The location of the neutral plane of the beam can be found (relative to the bottom of the laminate) using the equivalent area method:

$$
z_{n_{x}}=\frac{E_{s_{x}} t_{s}^{2}+E_{p_{x}}\left(t_{p}^{2}+2 t_{p} t_{s}\right)}{2\left(E_{s_{x}} t_{s}+E_{p_{x}} t_{p}\right)} .
$$

Next, it can be seen that the tensile and compressive forces above and below the neutral axis, respectively, are equal in magnitude but opposite in direction. Therefore, let $P_{x}=$ $P_{x}^{+}=-P_{x}^{-}$, the tensile and compressive force respectively causing the moment. Then, the moments per unit width acting on each end of the composite laminate plate (taken about the neutral axis) are

$$
\begin{aligned}
& M_{x}=M_{x}^{+}+M_{x}^{-}=P_{x}^{+}\left(\frac{t_{p}+t_{s}-z_{n_{x}}}{2}\right) \\
& +P_{x}^{-}\left(-\frac{z_{n_{x}}}{2}\right)=P_{x}\left(\frac{t_{p}+t_{s}}{2}\right)=M_{p_{x}}+M_{s_{x}}
\end{aligned}
$$

or, by also manipulating (16),

$$
P_{x}\left(\frac{t_{p}+t_{s}}{2}\right)=M_{p_{x}}\left(1+\frac{E_{s_{x}} I_{s_{x}}}{E_{p_{x}} I_{p_{x}}}\right)
$$

Lastly, the total strain experienced by both lamina (MFC and laminate substrate) at the interface boundary is the same. Hence, at the interface,

$$
\frac{T_{p_{x}}}{E_{p_{x}}}-\nu_{p_{x y}} \frac{T_{p_{y}}}{E_{p_{x}}}+d_{33} \frac{V}{x_{e}}=-\left(\frac{T_{s_{x}}}{E_{s_{x}}}-\nu_{p_{x y}} \frac{T_{s_{y}}}{E_{s_{x}}}\right),
$$


where $T_{(\cdot)}$ is a combination of direct stress (caused by the linear piezoelectric actuation) and bending stress (due to the resulting couple). However, for application to a beam the transverse strain terms can be neglected. This then expands to

$$
\begin{aligned}
\frac{1}{E_{p_{x}}}\left[\frac{P_{x}}{A_{p_{x}}}+E_{p_{x}} \kappa_{x}\right. & \left.\left(-\frac{t_{p}}{2}\right)\right]+d_{33} \frac{V}{x_{e}} \\
& =-\frac{1}{E_{s_{x}}}\left[\frac{P_{x}}{A_{s_{x}}}+E_{s_{x}} \kappa_{x}\left(\frac{t_{s}}{2}\right)\right]
\end{aligned}
$$

or

$$
P_{x}\left(\frac{1}{E_{p_{x}} A_{p}}+\frac{1}{E_{s_{x}} A_{s}}\right)+\kappa_{x}\left(\frac{t_{s}+t_{p}}{2}\right)=-d_{33} \frac{V}{x_{e}} .
$$

Equation (20) is essentially the balance of internal strains due to axial forces with the external strain from the MFC actuator, which is given by the term $d_{33} V / x_{e}$. The piezoelectric strain term is negative due to the sign convention adopted (hogging corresponding with negative curvature). Substituting $P_{x}$ from (19) into (20), and also noting $M_{p_{x}}=E_{p_{x}} I_{p_{x}} \kappa_{x}$, the curvature in the $x$ axis may be written as (21). Also note that by expressing the terms $I_{(\cdot)}$ and $A_{(\cdot)}$ in the curvature equation (21) in terms of the width and the thickness of each material the curvature can be written in terms of the elastic moduli and thickness only (see Equation 22).

Equations (21) and (22) imply the change in curvature is linear with increasing piezoelectric strain. If the piezoelectric coefficient, $d_{33}$ (and, hence, $\alpha_{x}$ ) were treated as constant the curvature (and hence deflection) change would be a simple linear scale of the excitation voltage, $V$. In reality, $d_{33}$ varies with the input voltage as discussed previously and (21) or (22) can be written explicitly in terms of $V$ using the fitted model of (9).

The exact same procedure (effectively Equation 22) may be used to obtain $\kappa_{y}$ and, hence, $M_{y}$. However, although the substrate in this case is isotropic the MFC is not, and $E_{p_{y}}<E_{p_{x}}$ which causes the neutral axis in the $y z$ to shift downwards. Also, the width for the bending in the $y$ axis is $L_{p}$. Using both $\kappa_{x}$ and $\kappa_{y}$, the moments generated at the ends of the actuator plate may then be found from (12) and (13). The deflection of such a composite plate, simply supported at the corner points, may then be given by plate theory (Timoshenko and Woinowsky-Kreiger 1959):

$$
\begin{aligned}
\delta(x, y)=\frac{\left(M_{x}-\nu_{x y} M_{y}\right)}{D_{x}}\left(\frac{L_{s}}{2} x-\frac{x^{2}}{2}\right) & \\
+ & +\frac{\left(M_{y}-\nu_{y x} M_{x}\right)}{D_{y}}\left(\frac{w_{s}}{2} y-\frac{y^{2}}{2}\right) .
\end{aligned}
$$

Since the moments in each plane are in the opposite direction, a classical anticlastic surface results (Figure 13).

\section{Encastre composite plate bending model (unequal laminate and actuator lengths)}

Now, let the length of the laminate exceed that of the actuator. The mounting of the laminate is also the encastre configuration with both ends fixed (see Figure 14). In this case, the comparative thickness of the actuator patch to the laminate plate is not negligible so should not be neglected. The resulting plate has partially non-uniform sections, with a different thickness and flexural rigidity for the parts with the actuator patches. A formal solution to this is required a complete two dimensional consideration of the deformation. Instead, assume the actuator patch section (section 2) extends the width of the laminate. Now, the longitudinal bending may be treated as a pseduo-beam which reduces the complexity of the problem. The flexural stiffness may be modified in the usual way to account for the width of the laminate and the effect of transverse curvature. Note that the laminate plate is symmetric about the centreline, with two actuators in parallel. Therefore, in calculating the plate flexural rigidity, $D_{s_{x}}$, and second moment of area, $I_{s_{x}}$, only half the laminate's width is considered (i.e. $w_{s}=124$ $\mathrm{mm}$ ) Also note that the moments are treated as vectors and not scalars, so the sign of their numeric value does not necessarily tally with the direction of the moments as shown in Figure 14. For example, the moment $M_{0}$ is counterclockwise such that, numerically, $M_{0}<0$.

This approach still leads to rather involved beam equations. These are detailed in Table 4. The problem can be simplified here by noting that this configuration is symmetric longitudinally, such that, $R_{0}=-R_{L}=0, M_{0}=$ $-M_{L}, M_{a}=-M_{b}$, and $a=L_{s}-b$. Also note that $b-a=$ $L_{p}$, such that

$$
a=\frac{L_{s}-L_{p}}{2} \quad \text { and } \quad b=\frac{L_{s}+L_{p}}{2} .
$$

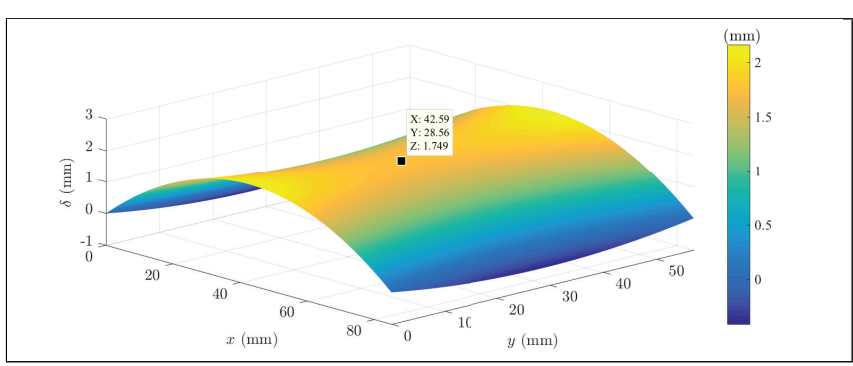

Figure 13. Anticlastic plate bending of MFC-substrate composite plate as a result of opposing orthogonal moments. Values for this example obtained by using data from Table 2. Data point shows values at centrepoint of plate. 


$$
\begin{aligned}
\kappa_{x} & =-\alpha_{x} V\left\{\frac{t_{s}+t_{p}}{2}+\frac{2}{t_{p}+t_{s}}\left[\frac{I_{p_{x}}}{A_{p_{x}}}\left(1+\frac{E_{p_{x}} A_{p_{x}}}{E_{s_{x}} A_{s_{x}}}\right)+\frac{I_{s_{x}}}{A_{s_{x}}}\left(1+\frac{E_{s_{x}} A_{s_{x}}}{E_{p_{x}} A_{p_{x}}}\right)\right]\right\}^{-1} \\
& =-\alpha_{x} V\left(\frac{6 E_{p_{x}} E_{s_{x}} t_{p} t_{s}\left(t_{p}+t_{s}\right)}{E_{p_{x}}^{2} t_{p}^{4}+4 E_{p_{x}} E_{s_{x}} t_{p}^{3} t_{s}+6 E_{p_{x}} E_{s_{x}} t_{p}^{2} t_{s}^{2}+4 E_{p_{x}} E_{s_{x}} t_{p} t_{s}^{3}+E_{s_{x}}^{2} t_{s}^{4}}\right) .
\end{aligned}
$$

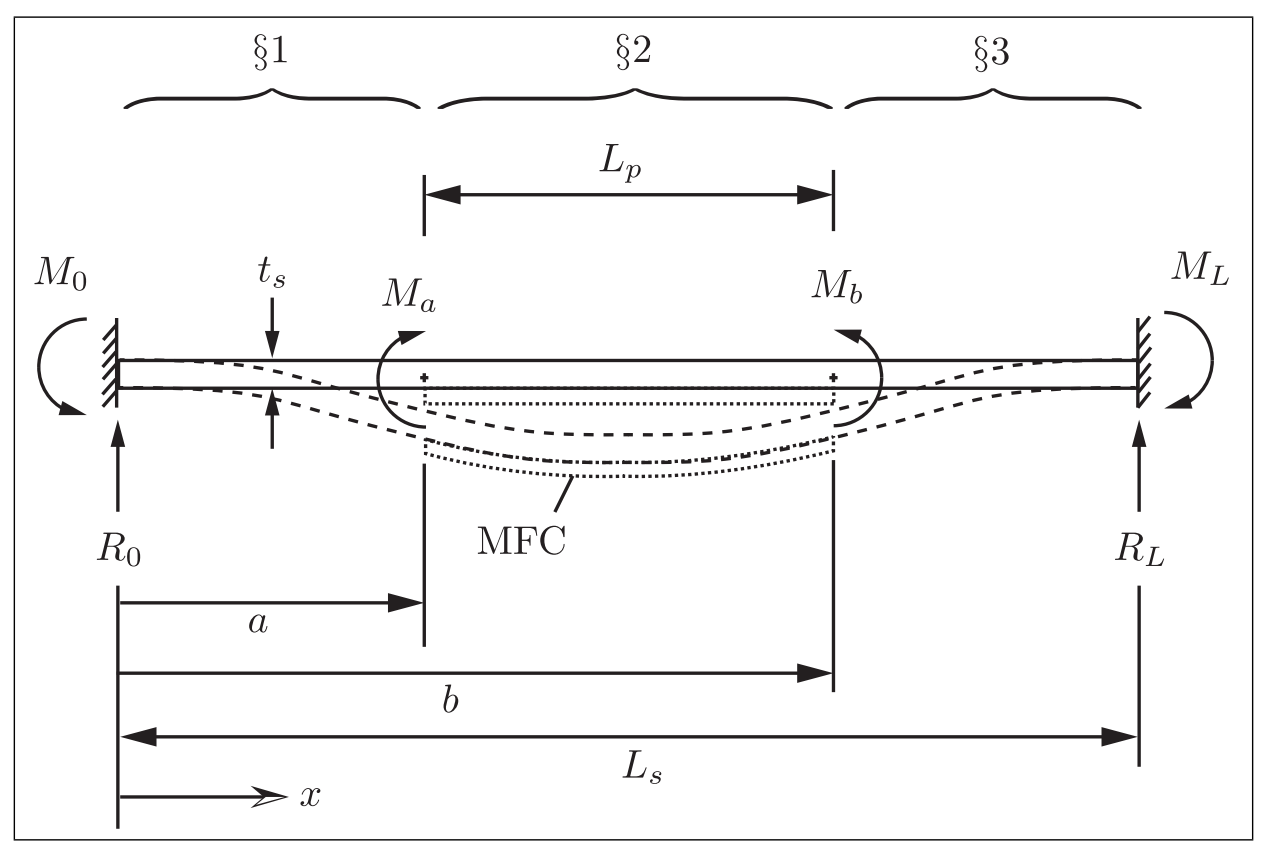

Figure 14. Diagram of the equivalent plate model for the laminate centreline. Section breaks in the uniformity of the beam are also shown.

The moments $M_{a}$ and $M_{b}$ are the effective bending moments generated at points $a$ and $b$ (corresponding with the ends of the MFC patch) due to the extension of the actuators. Since a moment is now transmitted through the laminate to the central composite section these moments are

$$
M_{a}=M_{x}-M_{0}
$$

and

$$
M_{b}=\left(-M_{x}\right)-M_{L}=-M_{x}+M_{0}
$$

The size of the reaction moments from the fixed ends, $M_{0}$ and $M_{L}=-M_{0}$ (for the symmetric case), will be proportional to the actuating moment and can be found by solving for one of the two boundary conditions at $L_{s}$ :

$$
\begin{gathered}
E_{3} I_{3} \frac{\partial \delta\left(L_{s}\right)}{\partial x}=0=M_{0} L_{s}+M_{a}\left(L_{s}-a\right)+M_{b}\left(L_{s}-b\right) \\
+\left[M_{0} b+M_{a}(b-a)\right]\left(\frac{E_{3} I_{3}}{E_{2} I_{2}}-1\right) \\
+M_{0} a\left(\frac{E_{3} I_{3}}{E_{1} I_{1}}-\frac{E_{3} I_{3}}{E_{2} I_{2}}\right)
\end{gathered}
$$

or

$$
\begin{gathered}
E_{3} I_{3} \delta\left(L_{s}\right)=0=M_{0} \frac{L_{s}^{2}}{2}+M_{a} \frac{\left(L_{s}-a\right)^{2}}{2}+M_{b} \frac{\left(L_{s}-b\right)^{2}}{2} \\
+\left[M_{0} b+M_{a}(b-a)\right]\left(\frac{E_{3} I_{3}}{E_{2} I_{2}}-1\right)\left(L_{s}-b\right) \\
+M_{0} a\left(\frac{E_{3} I_{3}}{E_{1} I_{1}}-\frac{E_{3} I_{3}}{E_{2} I_{2}}\right)\left(L_{s}-b\right) \\
+\left[M_{0} \frac{b^{2}}{2}+M_{a} \frac{(b-a)^{2}}{2}\right]\left(\frac{E_{3} I_{3}}{E_{2} I_{2}}-1\right) \\
\quad+M_{0} a\left(\frac{E_{3} I_{3}}{E_{1} I_{1}}-\frac{E_{3} I_{3}}{E_{2} I_{2}}\right)(b-a) \\
+M_{0} \frac{a^{2}}{2}\left(\frac{E_{3} I_{3}}{E_{1} I_{1}}-\frac{E_{3} I_{3}}{E_{2} I_{2}}\right)
\end{gathered}
$$

Equations (23) through (26) can be solved for $M_{a}, M_{b}$, and $M_{0}$ in terms of the plate geometries $\left(a, b\right.$, and $\left.L_{s}\right)$, the flexural rigidity of each section, and $M_{x}$ which may be computed by (12). The solutions are more convoluted than the starting equations, and are best solved using symbolic computation (e.g. MATLAB symbolic toolbox). Using the material property values for the MFC and laminate substrate (Table 2) the deflection for each section is solved for the operating voltage range and plotted in Figure 15. 
Table 4. Encastre beam deflection equations

For the configuration shown in Figure 14, the bending equations for each section, with the origin at $x=0$, are as follows:

Section $1(0 \leq x \leq a)$ :

$$
\begin{gathered}
E_{1} I_{1} \frac{\partial^{2} \delta(x)}{\partial x^{2}}=M_{0}+R_{0} x, \quad E_{1} I_{1} \frac{\partial \delta(x)}{\partial x}=M_{0} x+R_{0} \frac{x^{2}}{2}+A_{1}, \\
E_{1} I_{1} \delta(x)=M_{0} \frac{x^{2}}{2}+R_{0} \frac{x^{3}}{6}+A_{1} x+A_{2} .
\end{gathered}
$$

Section $2(a \leq x \leq b)$ :

$$
\begin{gathered}
E_{2} I_{2} \frac{\partial^{2} \delta(x)}{\partial x^{2}}=M_{0}+R_{0} x+M_{a}, \quad E_{2} I_{2} \frac{\partial \delta(x)}{\partial x}=M_{0} x+R_{0} \frac{x^{2}}{2}+M_{a}(x-a)+A_{3}, \\
E_{2} I_{2} \delta(x)=M_{0} \frac{x^{2}}{2}+R_{0} \frac{x^{3}}{6}+M_{a} \frac{(x-a)^{2}}{2}+A_{3}(x-a)+A_{4} .
\end{gathered}
$$

Section $3\left(b \leq x \leq L_{s}\right)$ :

$$
\begin{aligned}
E_{3} I_{3} \frac{\partial^{2} \delta(x)}{\partial x^{2}} & =M_{0}+R_{0} x+M_{a}+M_{b} \\
E_{3} I_{3} \frac{\partial \delta(x)}{\partial x} & =M_{0} x+R_{0} \frac{x^{2}}{2}+M_{a}(x-a)+M_{b}(x-b)+A_{5}, \\
E_{3} I_{3} \delta(x) & =M_{0} \frac{x^{2}}{2}+R_{0} \frac{x^{3}}{6}+M_{a} \frac{(x-a)^{2}}{2}+M_{b} \frac{(x-b)^{2}}{2}+A_{5}(x-b)+A_{6} .
\end{aligned}
$$

Now, considering the boundary conditions at $x=0$ :

$$
E_{1} I_{1} \frac{\partial \delta(0)}{\partial x}=0 \quad \therefore A_{1}=0 \quad \text { and } \quad E_{1} I_{1} \delta(0)=0 \quad \therefore A_{2}=0 .
$$

At $x=a$,

$$
\frac{1}{E_{1} I_{1}}\left\{M_{0} a+R_{0} \frac{a^{2}}{2}\right\}=\frac{1}{E_{2} I_{2}}\left\{M_{0} a+R_{0} \frac{a^{2}}{2}+A_{3}\right\} . \quad \therefore A_{3}=\left(M_{0} a+R_{0} \frac{a^{2}}{2}\right)\left(\frac{E_{2} I_{2}}{E_{1} I_{1}}-1\right) .
$$

and

$$
\frac{1}{E_{1} I_{1}}\left\{M_{0} \frac{a^{2}}{2}+R_{0} \frac{a^{3}}{6}\right\}=\frac{1}{E_{2} I_{2}}\left\{M_{0} \frac{a^{2}}{2}+R_{0} \frac{a^{3}}{6}+A_{4} \cdot\right\} \quad \therefore A_{4}=\left(M_{0} \frac{a^{2}}{2}+R_{0} \frac{a^{3}}{6}\right)\left(\frac{E_{2} I_{2}}{E_{1} I_{1}}-1\right) .
$$

At $x=b$,

$$
\begin{gathered}
\frac{1}{E_{2} I_{2}}\left\{M_{0} b+R_{0} \frac{b^{2}}{2}+M_{a}(b-a)+\left(M_{0} a+R_{0} \frac{a^{2}}{2}\right)\left(\frac{E_{2} I_{2}}{E_{1} I_{1}}-1\right)\right\}=\frac{1}{E_{3} I_{3}}\left\{M_{0} b+R_{0} \frac{b^{2}}{2}+M_{a}(b-a)+A_{5}\right\} . \\
\therefore A_{5}=\left[M_{0} b+R_{0} \frac{b^{2}}{2}+M_{a}(b-a)\right]\left(\frac{E_{3} I_{3}}{E_{2} I_{2}}-1\right)+\left(M_{0} a+R_{0} \frac{a^{2}}{2}\right)\left(\frac{E_{3} I_{3}}{E_{1} I_{1}}-\frac{E_{3} I_{3}}{E_{2} I_{2}}\right) .
\end{gathered}
$$

and

$$
\begin{gathered}
\frac{1}{E_{2} I_{2}}\left\{M_{0} \frac{b^{2}}{2}+R_{0} \frac{b^{3}}{6}+M_{a} \frac{(b-a)^{2}}{2}+\left(M_{0} a+R_{0} \frac{a^{2}}{2}\right)\left(\frac{E_{2} I_{2}}{E_{1} I_{1}}-1\right)(b-a)+\left(M_{0} \frac{a^{2}}{2}+R_{0} \frac{a^{3}}{6}\right)\left(\frac{E_{2} I_{2}}{E_{1} I_{1}}-1\right)\right\} \\
=\frac{1}{E_{3} I_{3}}\left\{M_{0} \frac{b^{2}}{2}+R_{0} \frac{b^{3}}{6}+M_{a} \frac{(b-a)^{2}}{2}+A_{6}\right\} . \\
\therefore A_{6}=\left[M_{0} \frac{b^{2}}{2}+R_{0} \frac{b^{3}}{6}+M_{a} \frac{(b-a)^{2}}{2}\right]\left(\frac{E_{3} I_{3}}{E_{2} I_{2}}-1\right)+\left(M_{0} a+R_{0} \frac{a^{2}}{2}\right)\left(\frac{E_{3} I_{3}}{E_{1} I_{1}}-\frac{E_{3} I_{3}}{E_{2} I_{2}}\right)(b-a) \\
+\left(M_{0} \frac{a^{2}}{2}+R_{0} \frac{a^{3}}{6}\right)\left(\frac{E_{3} I_{3}}{E_{1} I_{1}}-\frac{E_{3} I_{3}}{E_{2} I_{2}}\right) .
\end{gathered}
$$


If it is assumed that the piezoelectric effect results in wholly bending strain (which is a reasonable assumption for the scenario investigated in this work) then the strain (on the laminate surface) should correspond with the bending straincurvature equation for plates. Therefore, we may write

$$
S(x)=-\frac{\left(-t_{s} / 2\right)}{\left(1-\nu_{x y} \nu_{y x}\right)}\left(\frac{M_{j}(x)}{E_{j} I_{j}}\right)
$$

where $M_{j}(x)$ is the total bending moment for each $j=$ $1,2,3$ section. For section 1 and 3 , the flexural rigidity is $E_{s} I_{s}$. For section 2, assuming the flexural rigidity of either the laminate or MFC-laminate composite will over/underestimate the curvature respectively. Therefore, an approximate middle-range value for the rigidity is taken, based on the fraction of the width covered by the actuator patch:

$$
E_{e} I_{e}=E_{s} I_{s}\left(\frac{w_{s}-w_{p}}{w_{s}}\right)+E_{x} I_{x}\left(\frac{w_{p}}{w_{s}}\right) .
$$

Since the variation in transverse curvature across the plate is not modeled, the moments in each beam section are constant, resulting in a step-wise variation in strain, dissimilar from the FE results. This, and assuming the strain is wholly bending-induced, are the main sources of error in this model.

\section{Uniform beam approximation}

Depending on the acceptable error, or when $t_{p} \ll t_{s}$, it may be convenient to neglect the actuator thickness. In doing so the rigidity ratios in the equations disappear and the bending moment may be equivalently written as

$$
M_{e}(x)=M_{0}+R_{0} x+M_{a}\langle x-a\rangle^{0}+M_{b}\langle x-b\rangle^{0}
$$

where the Macaulay brackets, e.g. $\langle x-a\rangle$, denote the ramp function

$$
\langle x-a\rangle^{n}= \begin{cases}0 & \text { if } x<a \\ (x-a)^{n} & \text { if } x \geq a\end{cases}
$$

Direct integration then gives the deflection, $\delta(x)$, as

$$
\begin{aligned}
\delta(x)=\frac{1}{E_{e} I_{e}}\left(\frac{M_{0}}{2} x^{2}\right. & +\frac{R_{0}}{6} x^{3}+\frac{M_{a}}{2}\langle x-a\rangle^{2} \\
& \left.+\frac{M_{b}}{2}\langle x-b\rangle^{2}+c_{1} x+c_{2}\right) .
\end{aligned}
$$

The constants of integration resolve to zero due to the fixed ends of the laminate, whilst evaluating the boundary conditions at $x=L_{s}$ leads to

$$
\begin{aligned}
M_{0}=\frac{3 M_{a}}{L^{2}} & {\left[\frac{2}{3} L_{s}\left(L_{s}-a\right)-\left(L_{s}-a\right)^{2}\right] } \\
& +\frac{3 M_{b}}{L^{2}}\left[\frac{2}{3} L_{s}\left(L_{s}-b\right)-\left(L_{s}-b\right)^{2}\right]
\end{aligned}
$$

and

$$
\begin{aligned}
R_{0}=\frac{6 M_{a}}{L_{s}^{3}}\left[\left(L_{s}-a\right)^{2}-L_{s}\left(L_{s}-a\right)\right] \\
\quad+\frac{6 M_{b}}{L^{3}}\left[\left(L_{s}-b\right)^{2}-L_{s}\left(L_{s}-b\right)\right]
\end{aligned}
$$

The effective moments $M_{a}$ and $M_{b}$ may be determined as before except that, as $t_{p} \rightarrow 0, M_{x} \rightarrow M_{s_{x}}$. Equation (30) then reduces to simply $M_{0}=-M_{a} L_{p} / L_{s}$. Then,

$$
\begin{gathered}
M_{0}=-M_{s_{x}}\left(\frac{L_{p}}{L_{s}-L_{p}}\right), \quad M_{a}=M_{s_{x}}\left(\frac{L_{s}}{L_{s}-L_{p}}\right), \\
\text { and } \quad M_{b}=-M_{s_{x}}\left(\frac{L_{s}}{L_{s}-L_{p}}\right)
\end{gathered}
$$

where $M_{s_{x}}=\kappa_{x} D_{s_{x}}+\nu_{s_{x y}} \kappa_{y} D_{s_{y}}$. The deflection can then be written as

$$
\begin{array}{r}
\delta(x)=-\frac{M_{s_{x}}}{E_{e} I_{e}}\left\{\left(\frac{L_{p}}{L_{s}-L_{p}}\right) \frac{x^{2}}{2}-\left(\frac{L_{s}}{L_{s}-L_{p}}\right) \frac{\langle x-a\rangle^{2}}{2}\right. \\
\left.+\left(\frac{L_{s}}{L_{s}-L_{p}}\right) \frac{\langle x-b\rangle^{2}}{2}\right\}
\end{array}
$$

Maximum deflection for the symmetric case then occurs at the centre of the laminate, it being

$$
\begin{aligned}
\delta^{\prime}=\delta\left(L_{s} / 2\right) \\
=-\frac{M_{s_{x}}}{2 E_{e} I_{e}}\left\{\left(\frac{L_{p}}{L_{s}-L_{p}}\right) \frac{L_{s}^{2}}{4}-\left(\frac{L_{s}}{L_{s}-L_{p}}\right)\left\langle\frac{L_{s}}{2}-a\right\rangle^{2}\right. \\
\left.\quad+\left(\frac{L_{s}}{L_{s}-L_{p}}\right)\left\langle\frac{L_{s}}{2}-b\right\rangle^{2}\right\} \\
=-\frac{M_{s_{x}}}{8 E_{e} I_{e}}\left\{\left(\frac{L_{p} L_{s}^{2}}{L_{s}-L_{p}}\right)-\left(\frac{L_{p}^{2} L_{s}}{L_{s}-L_{p}}\right)\right\} \\
=-\frac{M_{s_{x}}}{8 E_{e} I_{e}}\left(\frac{L_{p} L_{s}^{2}-L_{p}^{2} L_{s}}{L_{s}-L_{p}}\right) .
\end{aligned}
$$

The estimated strain is now

$$
S(x)=-\frac{\left(-t_{s} / 2\right)}{\left(1-\nu_{s_{x y}} \nu_{s_{y x}}\right)}\left(\frac{M_{e}(x)}{E_{e} I_{e}}\right) .
$$

The estimated deflections from this model are compared to the output from the non-uniform beam model in Figure 16. The parameters $S^{\prime}$ and $\delta^{\prime}$ refer to the strain and deflection, respectively, at the centre point of the laminate. This is where 


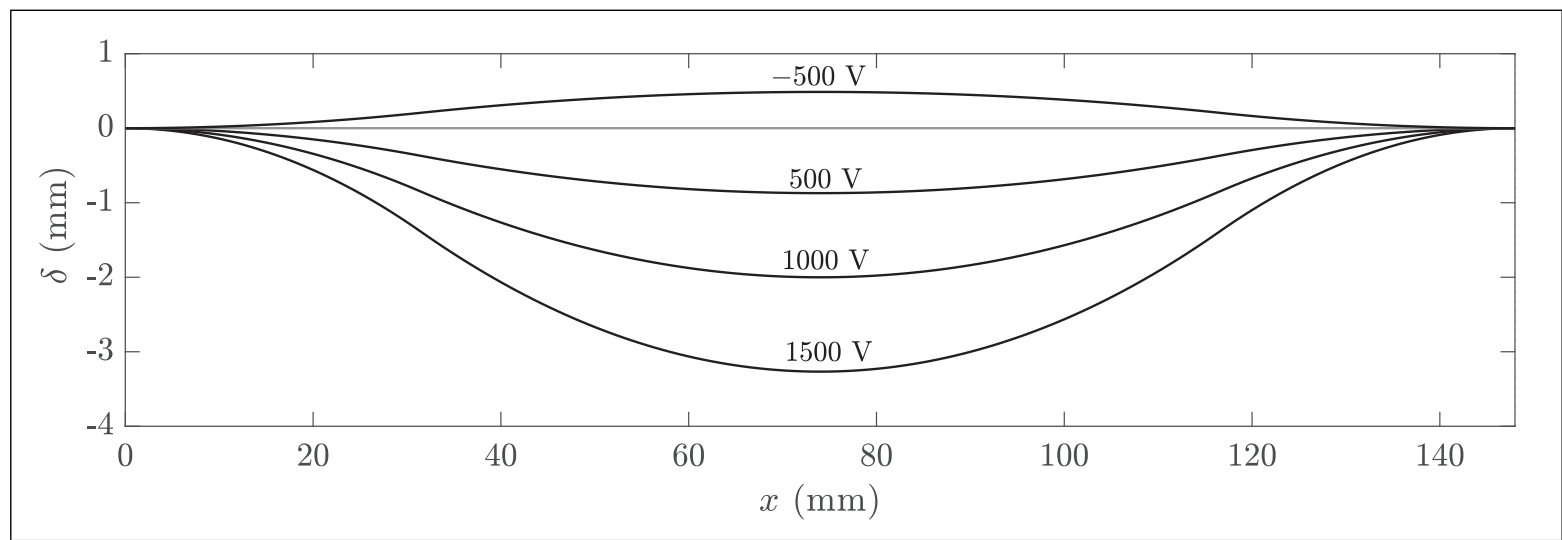

Figure 15. Deflection profiles from the bending beam model.

the difference in the two modeling approaches is greatest. The error increases with the amount for deflection but even at $1500 \mathrm{~V}$ only varies by $0.14 \mathrm{~mm}$. The variation in strain corresponding to this is in the order of $100 \mathrm{ppm}$.

\section{Results and discussion}

\section{Experimental results}

Experimental measurement of the strain on the laminate surface was carried out to collect data for validation of the modeling approaches. Two strain gauges, each arranged in a quarter-bridge configuration, were attached with standard cyanoacrylate ('super glue') to the centre and lateral areas of the composite as shown in Figure 17. The E-Glass laminate was placed in a support that fixed the movement of both ends, as per the setup of Figure 3, with the free length of the laminate at $148 \mathrm{~mm}$. Standard twisted copper leads $(1.5 \mathrm{kV}$ rated) delivered the excitation load from a TREK high voltage power amplifier $(-500$ to $1500 \mathrm{~V})$ driven by a bespoke variable source $(-2.5$ to $7.5 \mathrm{~V} \mathrm{DC})$. Strain measurements were taken at the centre only using a Measurements Group SB-10 switch and balance unit. The strain measurements were zeroed in the absence of the electric field. A more accurate means of measuring the laminate deflection was not available so a mechanical rule was instead used for reference.

The measured strain of the laminate for voltages across the operating range are shown in Figure 18a. Results only for an ascending monotonic change in voltage are shown. Repeatability in the strain output (and, hence, deflection) is consistently high. The strain per volt response increases at higher electric fields and is described well with a quadratic formula. The non-zero strain response at zero volts is interesting and indicates the presence of a bias due to the electric field that is not present in its absence. It is useful to illustrate here but in subsequent analysis the strain datum will be set to zero volts in the presence of the electric field. This makes it easier for comparison with the analytical and FE model results. The range of deflection amounted to approximately $4 \mathrm{~mm}$ through observation against the reference mechanical rule.

\section{Comparison of results}

Figure 19 compares the results from the analytical and finite element models with the experimental results. The agreement between all three is extremely good in terms of the strain measurements, with no more than 20 ppm difference across the operating range. The agreement between the estimated deflection of the analytical and finite element models is also very good. The trend in the deflection also agrees with the coarse observations made during the experiment. The deflection profile (along the $x$ axis) also agrees reasonably well between the analytical and numerical models (Figure 20). The differences between both models scale and begin to be noticeable from $1000 \mathrm{~V}$, where the analytical model bending is slightly more accentuated about the point of contraflexure (being the ends of the actuator patches; $a=31.5 \mathrm{~mm}$ and $b=116.5 \mathrm{~mm}$, respectively). This discrepancy is explained by the lack of modeling the (minor) variation in transverse curvature in the analytical model but perhaps more so by neglecting the linear strain caused by the end constraints. Another possibility is that some of the solid elements in the $\mathrm{FE}$ model locked. However, it was thought the size of the deflections experienced here would not make that a problem.. In any case, the maximum difference for $1500 \mathrm{~V}$ never exceeds $0.1 \mathrm{~mm}$. Of course, the small size of the errors in both FE and analytical models is, generally, due to the small size of the deflections experienced, as can be seen by the increasing growth rate in errors at the larger excitation voltages. The errors are reasonably acceptable up to the manufacturer recommended $1500 \mathrm{~V}$ excitation limit 


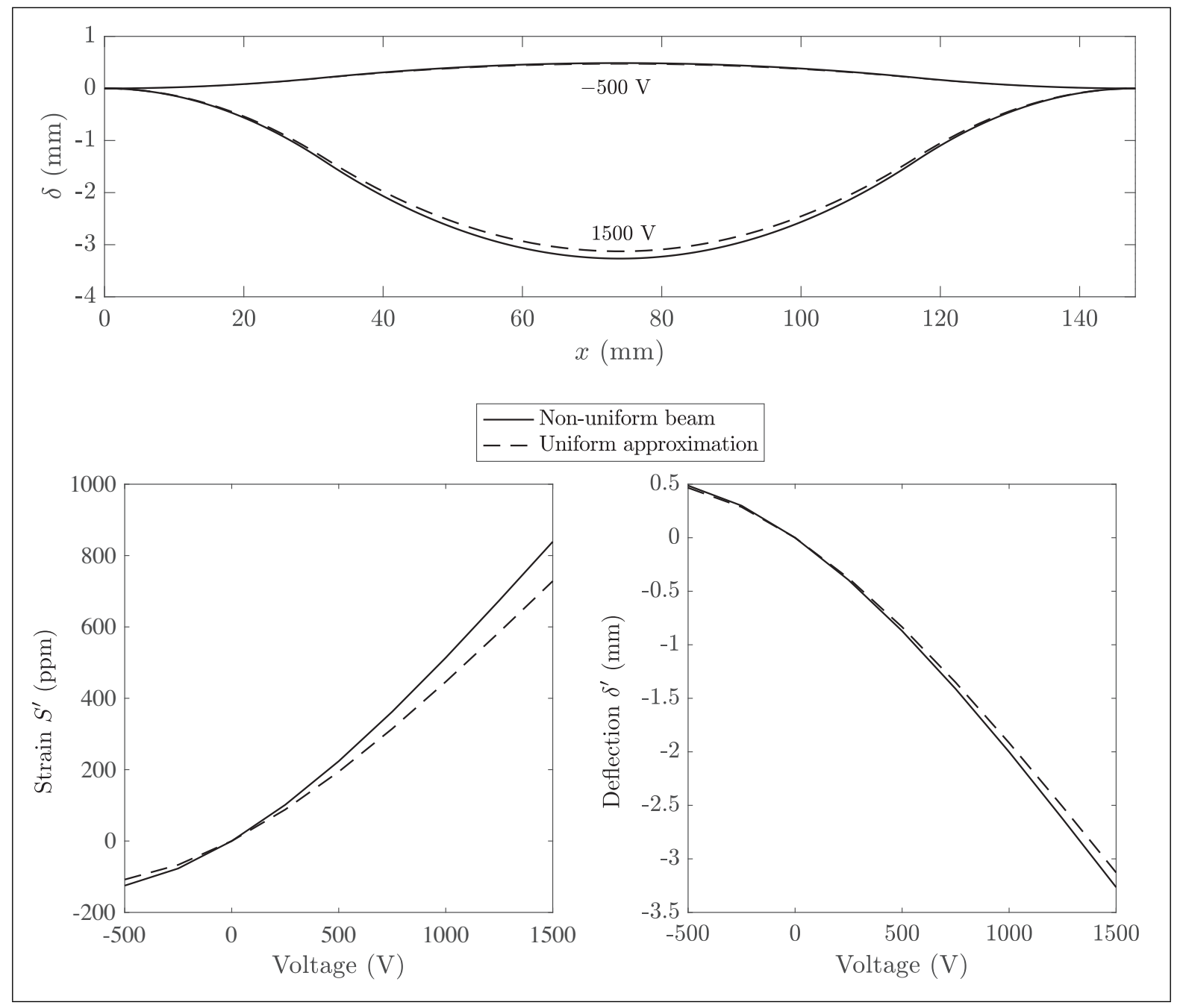

Figure 16. Error in uniform beam approximation model.

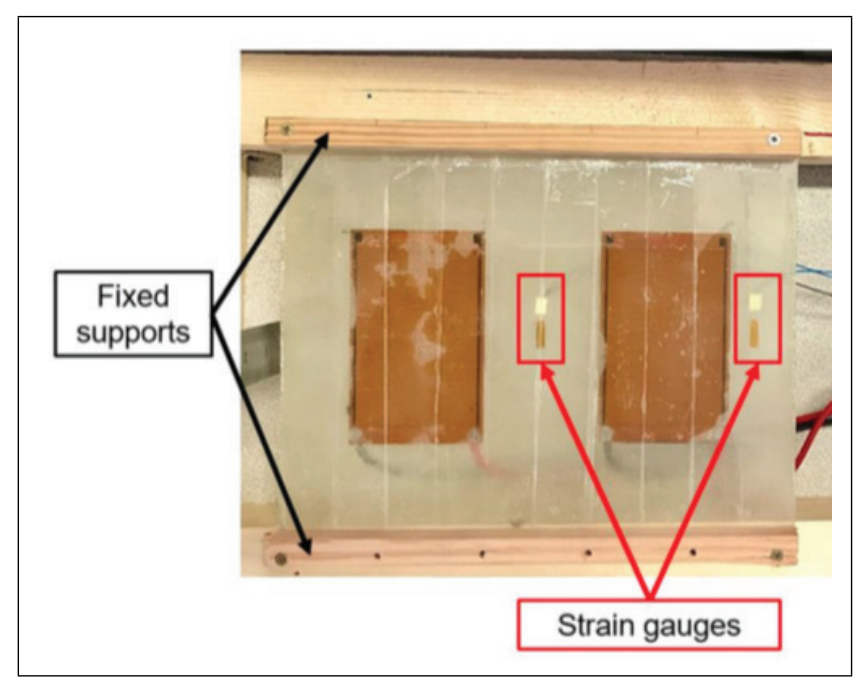

Figure 17. Experimental setup. MFCs and strain gauges mounted on the underside of the laminate when viewed from this image.

of operation, although some works have demonstrated reliable performance up to $1700 \mathrm{~V}$ (LaCroix and Ifju 2015). Refining the simplifications made in the analytical model would extend its use beyond the small deflection ranges experienced here.

Generally, for such small deflections, the maximum deflection at the centre of the sheet would be expected to be a linear response so the experimental results indicate the nonlinearity of the strain response of the actuator and not the structural deformation. This is modeled well with the nonlinear variation in $\alpha_{x}$ as evidenced by the very good agreement of both modeling approaches to the experimental measurements. Any nonlinear effects caused by the boundary constraints are therefore assumed negligible. However, this would not necessarily be the case if the laminate were significantly more flexible. As previously mentioned, it should be reasonable to approximate the response (in both strain and deflection) as linear over a range of about 0 to $1000 \mathrm{~V}$ without too much error. However, the nonlinear sensitivity in the higher voltage range, but more so the negative voltages, is quite evident. Therefore, unless the actuation system requires the full operational range of the MFC it would be simpler (both from an electrical systems 


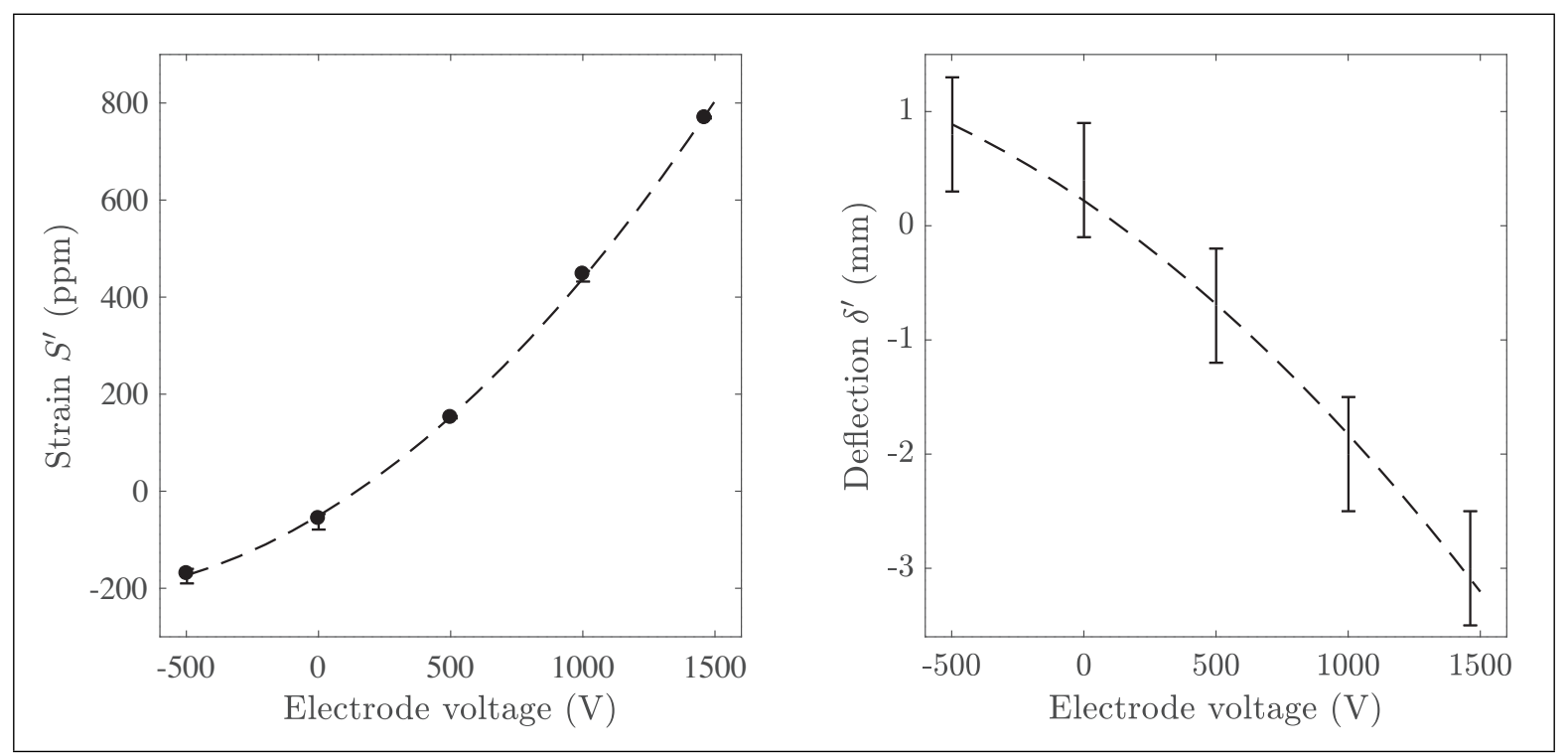

Figure 18. Centre point strain and estimated deflection of the encastre laminate. Strain was zeroed before application of the electric field, resulting in a non-zero response at $0 \mathrm{~V}$. For the experimentally-observed deflection, only the anticipated range is shown due to lack of precision in the measurement.

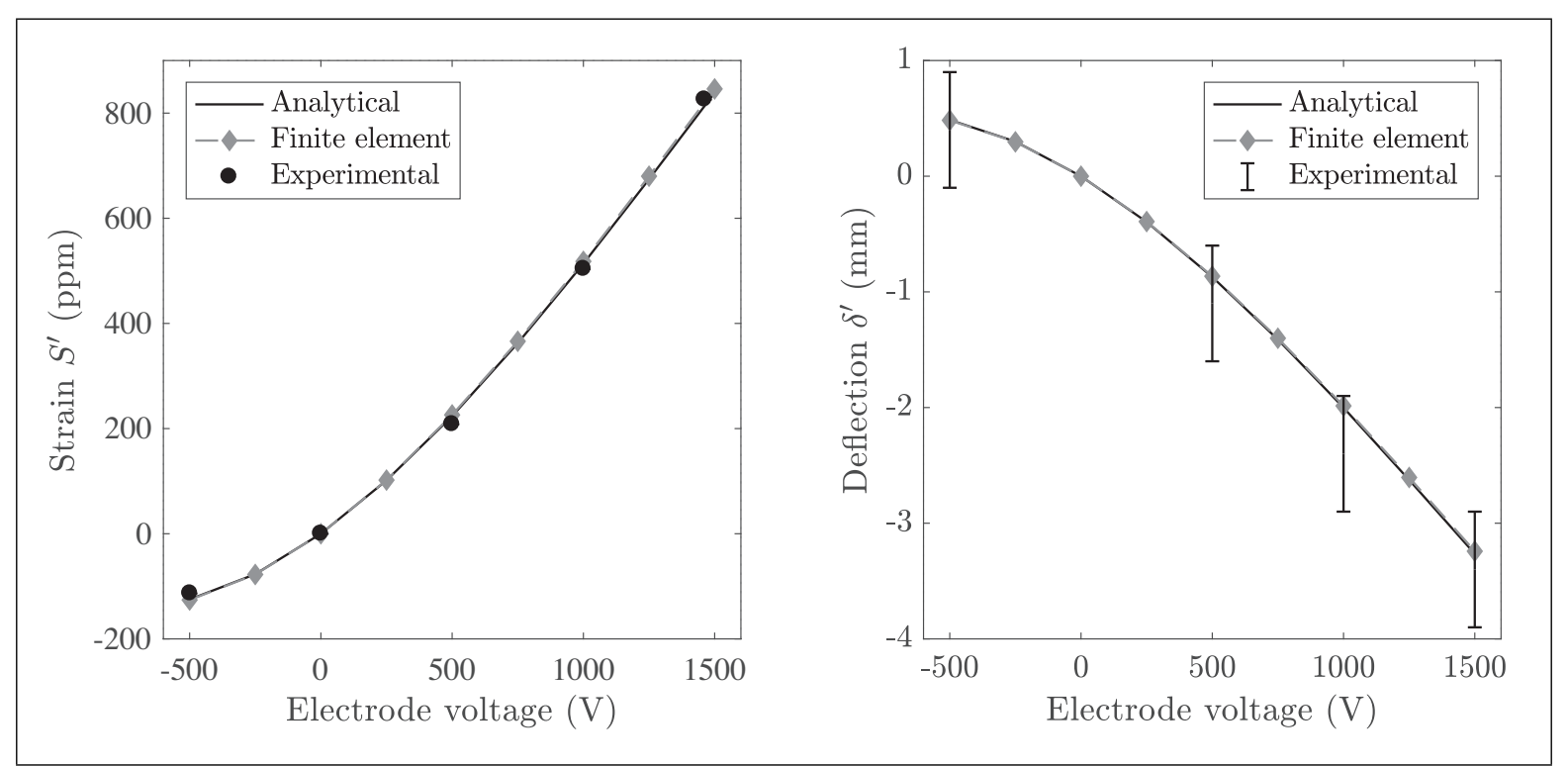

Figure 19. Comparison of analytical, finite element and experimental results at the laminate centre point. Values are referenced to zero strain at zero volts.

and modeling view) to operate only in the positive voltage range where a linear actuation response can be assumed. However, this limits the actuation to a single direction and having bidirectional motion may be desirable to maximise flexibility in the actuating structure.

The results also agree very well despite no temperature compensation on the strain measurements and a single resistance gauge used. This arrangement does however seem to be satisfactory for this experimental validation. It should also be noted that the modeled deflection in both the analytical or FE models did not take into account the sagging due to gravity acting on the laminate; this was expected to be very small and was shown to be the case in FE modeling, amounting to only $0.043 \mathrm{~mm}$ at the centrepoint.

The transverse variation in the deflection and strain (refer back to Figure 11), a result of using distributed actuator patches, is not ideal and complicates the analytical modeling. It was necessary to accommodate for this by averaging the beam rigidity. Despite this, the results were in very good agreement. However, the problem can be eliminated by increasing the density of actuator patches such that they extend across the entire width of the plate. Alternatively, the stiffness in the plate's normal $(z)$ axis would have to be increased. This could be achieved by using a stiffer resin matrix material or alternative fiber. 


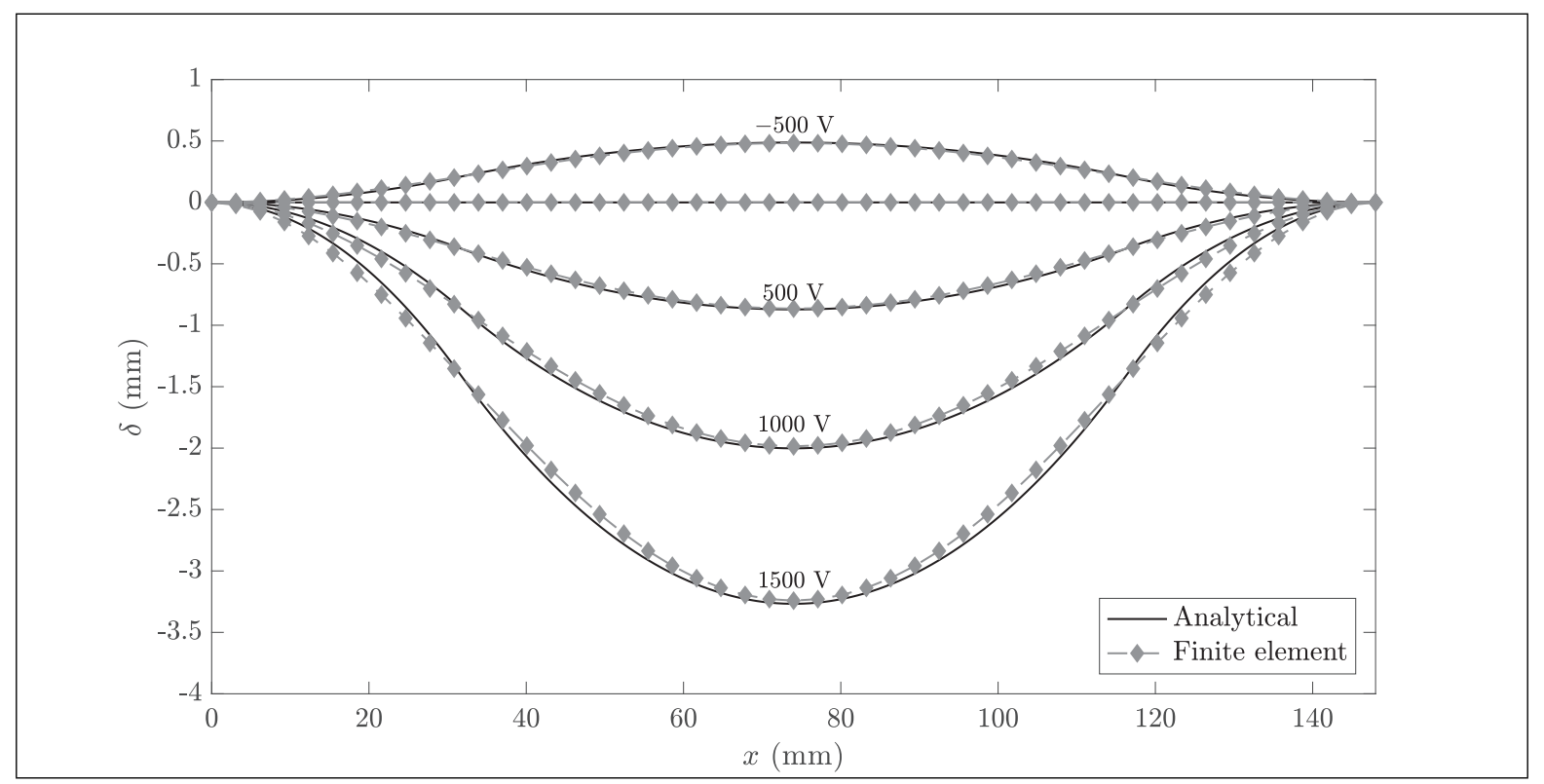

Figure 20. Comparison of centre-line deflection profiles from the analytical and finite element models.

It can also be noted that neither the FE nor analytical models accounted for the epoxy bond between the MFC and laminate. This is often neglected in studies because its thickness is much smaller than that of the laminate or MFC, but could therefore partially account for any minor differences in modeling results. The quality of the bond could also result in differences between experimental and simulation results.

\section{Deflection estimation from strain measurements}

In the absence of high-precision deflection measurements, a method of estimating it from the strain measurements was developed. This can be achieved with the bending model derived previously since the strain (and hence curvature) is already known.

Again, assuming the piezoelectric effect results in wholly bending strain the measured strain (taken at the centrepoint on the laminate's bottom surface) should correspond with the bending strain-curvature equation:

$$
S^{\prime}=S\left(L_{s} / 2\right)=-\frac{\left(-t_{s} / 2\right)}{\left(1-\nu_{x y} \nu_{y x}\right)}\left(\frac{M\left(L_{s} / 2\right)}{E_{e} I_{e}}\right)
$$

The bending moment at the centrepoint of the laminate is given by

$$
\begin{gathered}
M^{\prime}=M\left(L_{s} / 2\right)=M_{0}+M_{a}=M_{a}\left(1+\frac{M_{0}}{M_{a}}\right) \\
\therefore \frac{M_{a}}{E_{e} I_{e}}=\frac{M^{\prime}}{E_{e} I_{e}}\left(\frac{M_{a}}{M_{a}+M_{0}}\right) .
\end{gathered}
$$

Then, given (33),

$$
\begin{aligned}
\frac{M_{a}}{E_{x} I_{x}}= & \frac{M^{\prime}}{E_{e} I_{e}} \frac{E_{e} I_{e}}{E_{x} I_{x}}\left(\frac{M_{a}}{M_{a}+M_{0}}\right) \\
& =\frac{2 S^{\prime}\left(1-\nu_{x y} \nu_{y x}\right)}{t_{s}} \frac{E_{e} I_{e}}{E_{x} I_{x}}\left(\frac{M_{a}}{M_{a}+M_{0}}\right)
\end{aligned}
$$

The deflection at this point on the plate is (35). Combining (34) with (35) the centrepoint deflection can then be written as (36).

The estimated deflections from this model are plotted in Figure 21 and agree very well with the previous results. As anticipated, fixing the properties of the laminate makes the deflection entirely proportional to the strain. If $t_{p} \ll t_{s}$ then (36) can be reduced to

$$
\delta^{\prime}=-\frac{S^{\prime}\left(1-\nu_{x y} \nu_{y x}\right)}{4 t_{s}}\left(\frac{L_{p} L_{s}^{2}-L_{p}^{2} L_{s}}{L_{s}-L_{p}}\right) .
$$

The estimates for this uniform approximation are also shown in Figure 21. Again, the error scales with the amount of deflection, with the difference between both estimates reaching about $0.3 \mathrm{~mm}$ at $1500 \mathrm{~V}$.

Whilst the analytical model agrees very well with the FE model, the lack of precision in the experimentallymeasured deflection limits the degree of validation possible here. Confidence can be gleaned, however, from the fact that the experimental strain measurements are consistent and agree well with the FE and analytical models, and so should translate to a similar match in the experimental deflections. This is shown in the quality of the estimates from (36). Nevertheless, validation against more precise experimental deflection 


$$
\begin{gathered}
\delta\left(L_{s} / 2\right)=\frac{1}{E_{x} I_{x}}\left\{M_{0} \frac{L_{s}^{2}}{8}+\frac{M_{a}}{2}\left(\frac{L_{s}}{2}-a\right)^{2}+\left[M_{0} a\left(\frac{L_{s}}{2}-a\right)+M_{0} \frac{a^{2}}{2}\right]\left(\frac{E_{x} I_{x}}{E_{s} I_{s}}-1\right)\right\} \\
=\frac{M_{a}}{8 E_{x} I_{x}}\left\{\frac{M_{0}}{M_{a}} L_{s}^{2}+L_{p}^{2}+\frac{M_{0}}{M_{a}}\left[2 L_{p}\left(L_{s}-L_{p}\right)+\left(L_{s}-L_{p}\right)^{2}\right]\left(\frac{E_{x} I_{x}}{E_{s} I_{s}}-1\right)\right\} . \\
\delta^{\prime}=\frac{S^{\prime}}{4 t_{s}}\left(1-\nu_{x y} \nu_{y x}\right) \frac{E_{e} I_{e}}{E_{x} I_{x}} \frac{M_{a}}{\left(M_{a}+M_{0}\right)}\left\{\frac{M_{0}}{M_{a}} L_{s}^{2}+L_{p}^{2}+\frac{M_{0}}{M_{a}}\left[L_{p}^{2}\left(L_{s}-L_{p}\right)+\left(L_{s}-L_{p}\right)^{2}\right]\left(\frac{E_{x} I_{x}}{E_{s} I_{s}}-1\right)\right\} . \\
z / c=5(t / c)\left[0.2969 \sqrt{x / c}-0.1260(x / c)-0.3516(x / c)^{2}+0.2843(x / c)^{3}-0.1015(x / c)^{4}\right] . \\
\frac{\mathrm{d} z}{\mathrm{~d} x}=5(t / c)\left[0.1485(x / c)^{-1 / 2}-0.1260-0.7032(x / c)+0.8529(x / c)^{2}-0.406(x / c)^{3}\right] .
\end{gathered}
$$

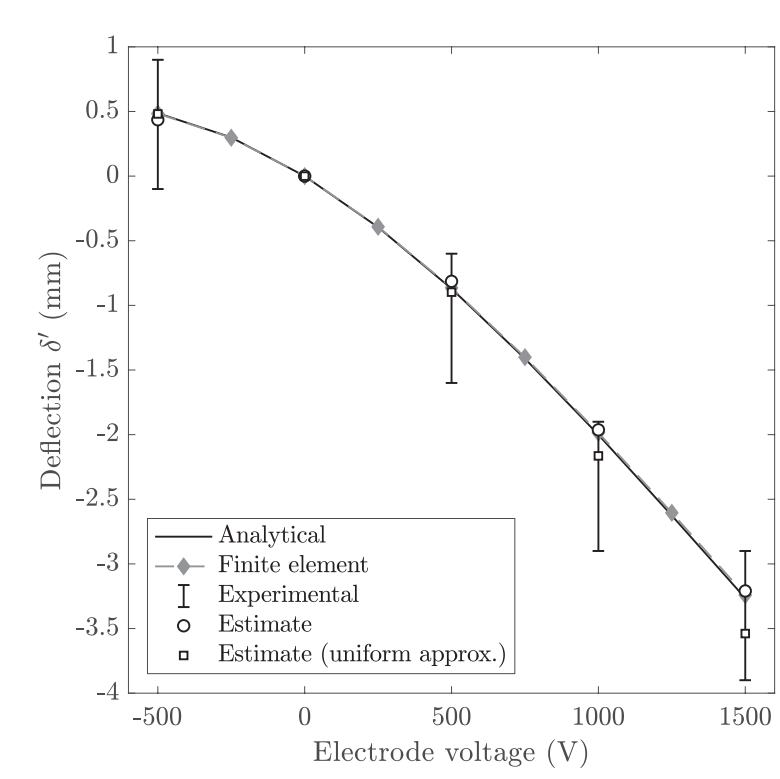

Figure 21. Estimates of centre-point deflection from experimental strain measurements.

measurements is left for future work, whereby a precision laser displacement sensor or digital image correlation setup should be used. Such investigation will also provide better validation of the transverse deflections observed in the FE model. Also, whilst element locking does not seem to have happened with the FE model, a comparison with a shell element model (or increasing the through-thickness mesh density) would provide further information on the accuracy of the FE model used.

\section{Application of analytical model}

Although the accuracy of the analytical approach was shown to be good compared to the FEA and experimental results, it is only an approximate and lacks the versatility of finite element modeling. The accuracy is also likely to deteriorate as the length and width dimensions become more disparate, as well as when the thicknesses increase. However, the utility of the analytical model is in providing a reasonably quick estimation of the deformation of two-dimensional structures. This is particularly useful for assessing the effects on aerofoils and fins, for which two-dimensional solutions to the pressure fields is achievable in relatively short time.

Consider the setup in Figure 22a in which an MFC patch is used to deform the bottom aft surface of a symmetric NACA 0010 aerofoil, having a chord length $c$. The deformable surface uses the same properties as the laminate previously derived. The MFC patch is located such that the deflection of the bottom-aft surface of the aerofoil may be modeled using the analytical beam model. For this setup the ratio $L_{p} / L_{s}=$ 0.5 , where $L_{s}=0.9 c$. Let $A$ and $B$ be the chordwise locations defining the ends of the morphing surface. Then, for the case in Figure 22a $A=0.1 c$ and $B=c$ where $A B \approx$ $L_{s}$.

The aerofoil thickness distribution is defined by (37). Differentiating this with respect to $x / c$ gives the surface slope profile as (38) where the thickness, $t / c$, is 0.1 . For the lower surface, negative versions of (37) and (38) apply. Since the slopes across the aft of the aerofoil (not including the leading edge) are small the beam deflection from the analytical model can be mapped simply to the aerofoil surface curvature with little error. Then

$(z / c)_{l}= \begin{cases}-z / c & \text { for } x / c \leq A \\ -z / c+(\delta / c) \cos \left(\frac{\mathrm{d} z}{\mathrm{~d} x}\right) & \text { for } A<x / c \leq B\end{cases}$ 


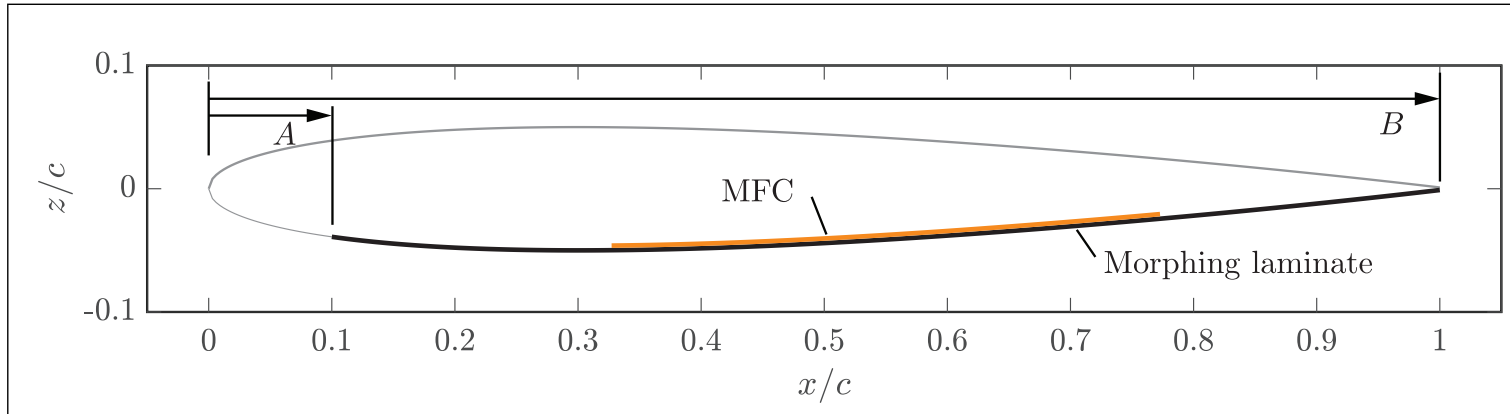

(a) Setup.

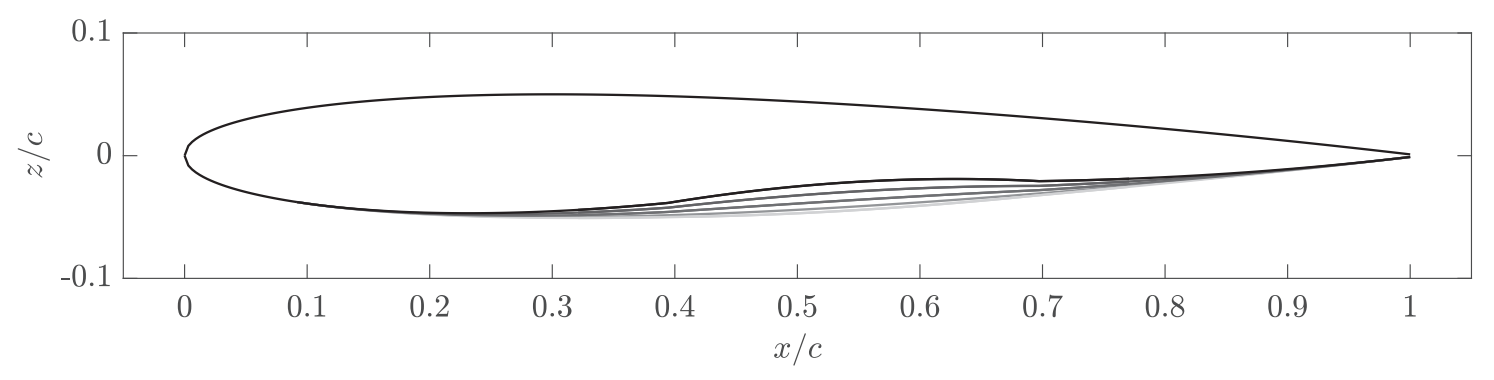

(b) Simulated morphing between -500 to $1500 \mathrm{~V}$.

Figure 22. modeled MFC-actuated NACA 0010 aerofoil.

Since the slope distribution is reasonably small (no more than -6.8 degrees at the trailing edge) the cosine transformation could be removed without noticeable error.

The resulting changes in the aerofoil surface, for the operating voltage range $(-500$ to $1500 \mathrm{~V})$ are shown in Figure 22b. Such a configuration is particularly suited for modifying the stall behaviour. This result is evidenced by importing the resulting aerofoil geometry into the XFOIL program. Figure 23 shows the pressure distribution across the aerofoil at a Reynolds number, Re, of $5 \times 10^{6}$. Shown are the pressure distributions for both the baseline NACA 0010 aerofoil, the same aerofoil with a flap deflected at 1 degree and hinged at $0.8 c$, and the MFC-actuated morphing aerofoil with $1500 \mathrm{~V}$ excitation voltage. The most noticeable effect in the case of the morphing aerofoil is the small pocket of increased pressure allowing for a minor recovery in the lift lost due to having a thick leading edge. This affords a minor increase in lift and a slight increase in the stall angle ( $\sim 1$ degree) over the original symmetric aerofoil and a conventional flapped aerofoil (see Figure 24a) in the example shown. The other benefit apparent in Figure 24a is a slight increase in the post-stall lift. However, the adverse pressure gradient that results from the morph trips the boundary layer into turbulent flow much earlier along the chord at lower angles of attack, resulting in an increase in drag. With a flapped aerofoil, although the pressure over the lower trailing region is slightly larger compared to the morphing aerofoil the flow over the upper surface in this region is already separated, ultimately reducing the available lift.

Whilst more pronounced effects on both lift and pitching moment can be achieved with comparatively small deflections of rigid flaps this modeling approach does not take into account the additional drag created by the gap between the flap and aerofoil. This has been shown to be in the region of a quarter to a third of the total drag on a typical wing (Cook 1971; Butler 1973; IHS 1992). Eliminating the gap and thus the excrescence drag of such a configuration and replacing it with contiguous morphing of a lifting structure is one of the main attractions for developing morphing wings, in addition to optimising the aerofoil shape for varying flight regimes. Further, the ability of the morphing foil to effect the pitching moment in a similar manner to a flap (shown in Figure 24b) has useful applications to changing the in-flight stability and trim characteristics without the aforementioned drag penalty.

Lastly, whilst the changes in aerodynamic characteristics shown here are marginal due to the limited deflection of the encastre configuration of the laminate, the analytical model can be reformulated for different boundary constraints to examine other configurations, such as a sliding constraint used by Debiasi et al. (2013a); Fichera et al. (2019). The use of the particular fiberglass laminate modeled in this paper would be limited to slow speeds as the low stiffness is unlikely to be able to resist deformation under larger 


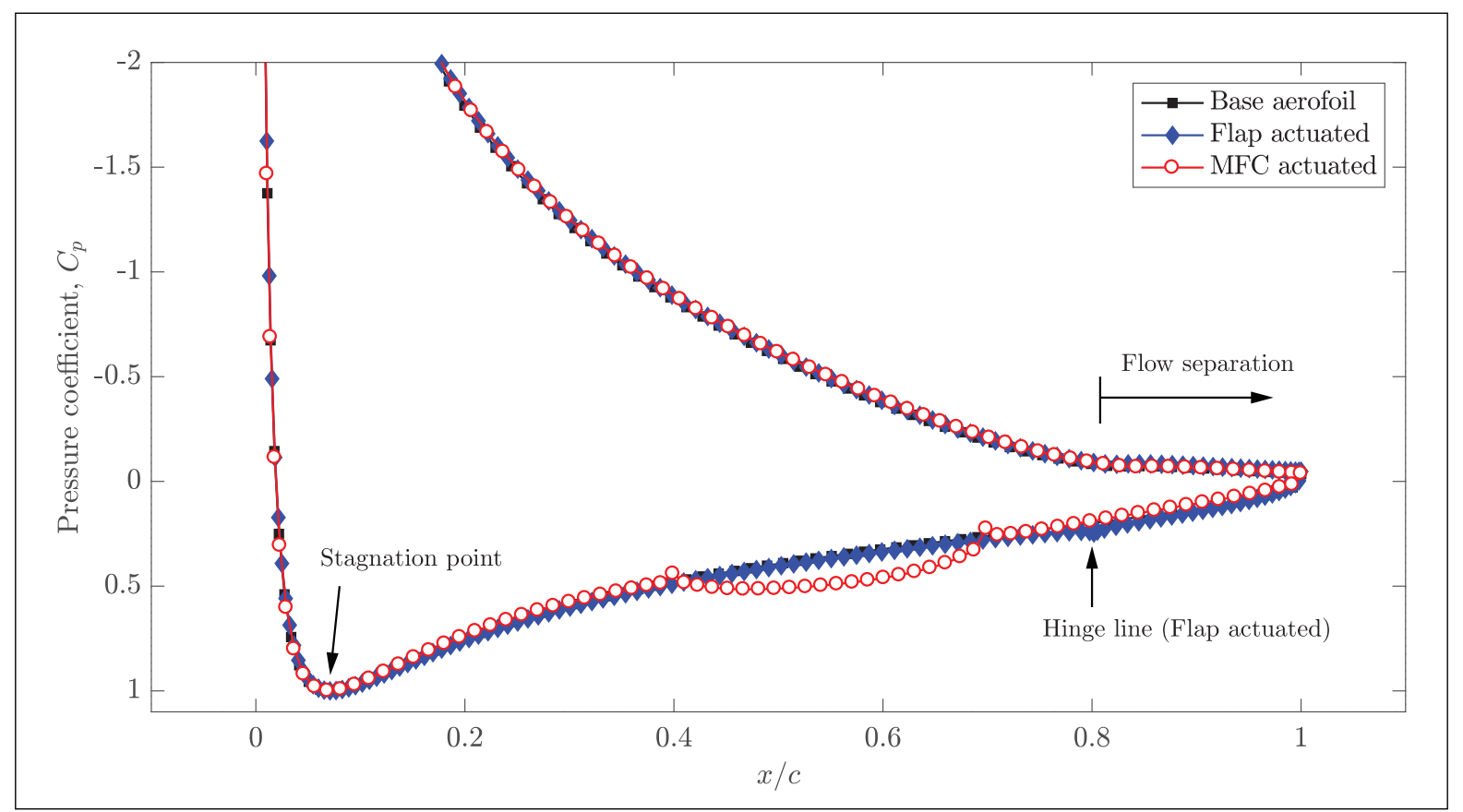

Figure 23. Pressure distribution for the NACA 0010 (base aerofoil) and different actuation types close to stall (18 degrees angle of attack) for $\operatorname{Re}=5 \times 10^{6}$. Flap actuation is shown for a 1 degree flap deflection with hinge at $0.8 \mathrm{c}$. MFC actuation is shown for 1500 $\mathrm{V}$ excitation voltage.

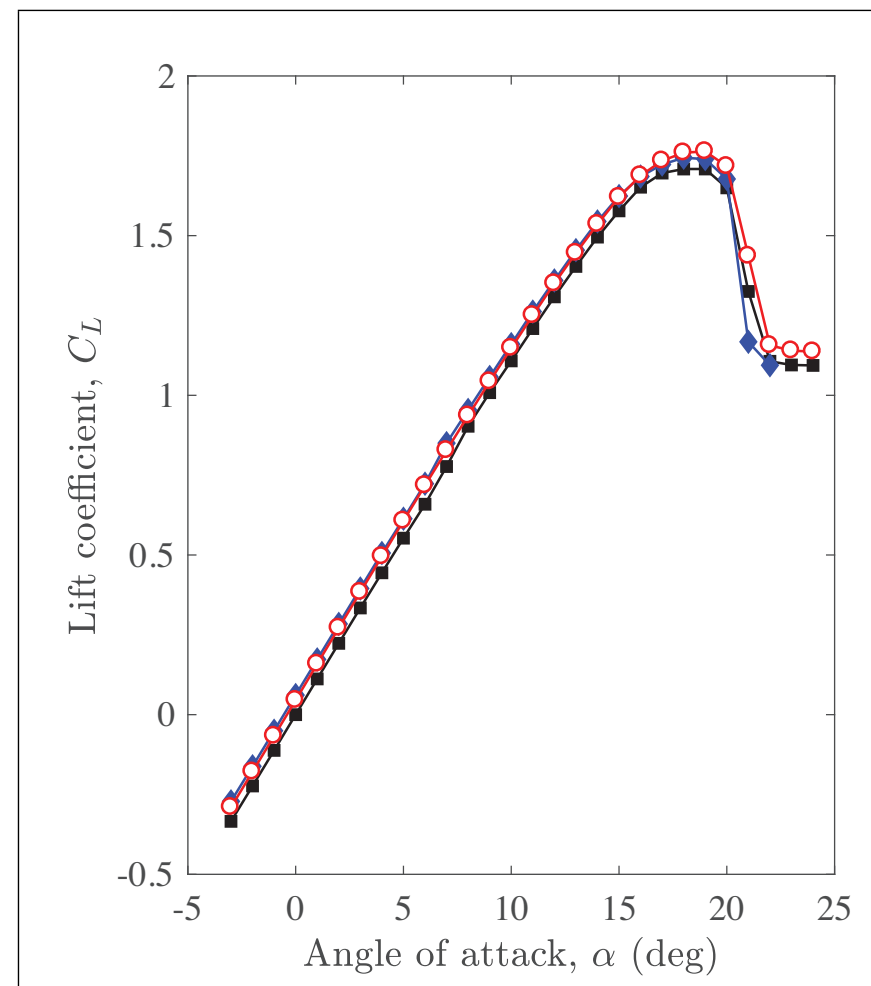

(a)

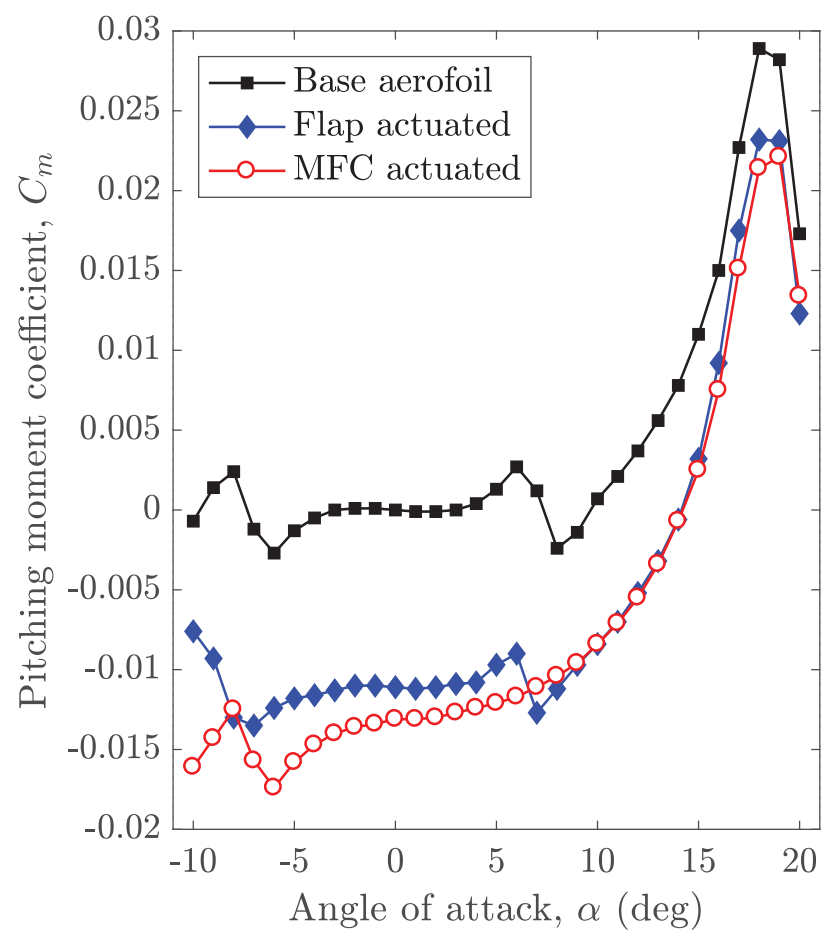

(b)

Figure 24. (a) Lift and (b) pitching moment coefficients for the NACA 0010 (base aerofoil) and different actuation types for Re $=5 \times 10^{6}$. Flap actuation is shown for a 1 degree flap deflection with hinge at $0.8 \mathrm{c}$. MFC actuation is shown for $1500 \mathrm{~V}$ excitation voltage.

aerodynamic loads. The stiffness would need to be larger to implement this setup on a larger, faster wing, whilst the amount of deflection can be increased by reducing its thickness.

\section{Conclusions}

This paper has presented the validation of two modeling approaches for MFC-actuated deflection of composite laminate plates. Excellent agreement between the two 
modeling approaches (a simplified plate bending model based on Euler-Bernoulli beam theory and a finite element approach using the traditional thermal analogy to piezoelectric strain) was demonstrated with experimental measurements of the bending strain at the centre-point of the laminate. Strains at the centre-point of the laminate were within $20 \mathrm{ppm}$ difference across both models and experimental measurements. Accurate deflection measurements of the laminate were not available, requiring a means to estimate the deflection from the experimental results. This method was also shown to give consistent results with the models, with differences not exceeding 0.1 $\mathrm{mm}$ at the centre-point. Overall differences in longitudinal deflection profiles between both modeling approaches were also within this tolerance. The usefulness of the analytical model was demonstrated on simulations of the aerodynamic changes due to varying the camber on a MFC-actuated morphing aerofoil.

\section{Declaration of conflicting interests}

The authors declare no potential conflicts of interest with respect to the research, authorship, and/or publication of this article.

\section{Funding}

This research was funded by a small research grant from the University of Hertfordshire School of Engineering \& Computer Science.

\section{References}

ANSI/IEEE (1987) Ieee standard on piezoelectricity. ANSI/IEEE Std 176-1987, New York, New York, USA.

Bilgen O (2010) Aerodynamic and Electromechanical Design, Modeling and Implementation of Piezocomposite Airfoils. $\mathrm{PhD}$ Thesis, Virginia Polytechnic Institute and State University, Blacksburg, Virginia, USA.

Bilgen O and Friswell MI (2012) Piezoceramic composite actuators for a solid-state variable- camber wing. In: ICAST2012: 23rd International Conference on Adaptive Structures and Technologies. Nanjing, China.

Bilgen O and Friswell MI (2013) Implementation of a continuousinextensible-surface piezocomposite airfoil. Journal of Aircraft 50(4): 508-518.

Bilgen O, Karami MA, Inman DJ and Friswell MI (2011) The actuation characterization of cantilevered unimorph beams with single crystal piezoelectric materials. Smart Materials and Structures 20: 1-9.
Bilgen O, Kochersberger KB and Inman DJ (2009) Macro-fiber composite actuators for a swept wing unmanned aircraft. The Aeronautical Journal 113(1144): 385-395.

Bilgen O, Kochersberger KB, Inman DJ and Ohanian OJ (2010) Novel, bidirectional, variable-camber airfoil via macro-fiber composite actuators. Journal of Aircraft 47(1): 303-314.

Bowen CR, Butler R, Jervis R, Kim HA and Salo AIT (2006) Morphing and shape control using unsymmetrical composites. Journal of Intelligent Material Systems and Structures 18(1): 89-98.

Bowen CR, Giddings PF, Salo AIT and Kim HA (2011) Modeling and characterization of piezoelectrically actuated bistable composites. IEEE Transactions on Ultrasonics, Ferroelectrics, and Frequency Control 58(9): 1737-1750.

Bowen CR, Kim HA and Salo AIT (2014) Active composites based on bistable laminates. Procedia Engineering 75: 140-144.

Bryant RG (2007) Overview of nasa langley's piezoelectric ceramic packaging technology and applications. In: 10th Japan International SAMPE Symposium and Exhibition. Tokyo, Japan.

Bu M, Melvin T, Ensell G, j S Wilkinson and Evans AGR (2003) Design and theoretical evaluation of a novel microfluidic device to be used for pcr. Journal of Micromechanics and Microengineering 13: S125-S130.

Butler SF (1973) Aircraft drag prediction for project appraisal and performance estimation. AGARD CP-124.

Chee CYK, Tong L and Steven GP (1998) A review on the modelling of piezoelectric sensors and actuators incorporated in intelligent structures. Journal of Intelligent Material Systems and Structures 9(1): 3-19.

Cook TA (1971) The effects of ridge excrescences and trailing-edge control gaps on twodimensional aerofoil characteristics. ARCRM-3698.

Côté F, Masson P, Mrad N and Cotoni V (2004) Dynamic and static modelling of piezoelectric composite structures using a thermal analogy with msc/nastran. Composite Structures 65(34): 4710-4484.

Crawley E and Anderson E (1990) Detailed models of piezoceramic actuation of beams. Journal of Intelligent Material Systems and Structures 1(1): 4-25.

Crawley E and DeLuis J (1987) Use of piezoelectric actuators as elements of intelligent structures. AIAA Journal 25(10): 13731385.

Debiasi M, Bouremel Y, Lu Z and Ravichandran V (2013a) Deformation of the upper and lower surfaces of an airfoil by macro fiber composite actuators. In: 31st AIAA Applied Aerodynamics Conference. San Diego, California, USA. 
Debiasi M, Leong CW and Bouremel Y (2013b) Application of macro-fiber-composite materials on uav wings. In: Proceedings of the Aerospace Technology Seminar (ATS) 2013. Singapore.

Deraemaeker A and Nasser H (2010) Numerical evaluation of the equivalent properties of macro fiber composite (mfc) transducers using periodic homogenization. International Journal of Solids and Structures 47(24): 3272-3285.

DeVoe DL and Pisano AP (1997) Modeling and optimal design of piezoelectric cantilever microactuators. Journal of Microelectromechanical Systems 6(3): 266-270.

Dwarakanathan D, Ramkumar R, Raja S and Subba Rao PS (2015) Design, development and ground testing of hingeless elevons for mav using piezoelectric composite actuators. Advances in Aircraft and Spacecraft Science 2(3): 303-328.

Fichera S, Isnardi I and Mottershead JE (2019) High-bandwidth morphing actuator for aeroelastic model control. Aerospace 6(2): 1-13.

Freed BD and Babuska V (1997) Finite element modeling of composite piezoelectric structures with msc/nastran. In: Proceedings of the SPIE 3041, Smart Structures and Materials 1997: Smart Structures and Integrated Systems.

Gao X, Shih WH and Shih Y (2009) Induced voltage of piezoelectric unimorph cantilevers of different nonpiezoelectric/piezoelectric length ratios. Smart Materials and Structures 18(125018): 1-8.

Gay D and Hoa SV (2003) Composite Materials: Design and Applications. CRC Press.

Giddings P, Bowen CR, Butler R and Kim HA (2008) Characterisation of actuation properties of piezoelectric bistable carbon-fibre laminates. Composites Part A: Applied Science and Manufacturing 39(4): 697-703.

Gustafson EA (2011) Design, Simulation, and Wind Tunnel Verification of a Morphing Airfoil. MS Thesis, Virginia Polytechnic Institute and State University, Blacksburg, Virginia, USA.

IHS (1992) Drag due to gaps round undeflected trailing-edge controls and flaps at subsonic speeds. ESDU 92039.

LaCroix BW and Ifju PG (2012) Finite element modeling of macro fiber composite piezoelectric actuators on micro air vehicles. In: 53rd AIAA/ASME/ASCE/AHS/ASC Structures, Structural Dynamics and Materials Conference. Honolulu, Hawaii, USA.

LaCroix BW and Ifju PG (2015) Investigating potential substrates to maximize out-of-plane deflection of piezoelectric macrofiber composite actuators. Journal of Intelligent Material Systems and Structures 26(7): 781-795.

Latalski J (2011) Modelling of mfc piezoelectric active elements in abaqus system. Eksploatacja i Niezawodnosc-Maintenance and Reliability 4: 72-78.

Lee AJ, Moosavian A and Inman DJ (2017) Control and characterization of a bistable laminate generated with piezoelectricity. Smart Materials and Structures 26(8).

Li L, Xue Z and Li C (2016) A modified pin force model for beams with active material bonded. Materials and Design 97: 249256.

Li X, Shih WY, Aksay IA and Shih WH (1999) Electromechanical behavior of pzt-brass unimorphs. Journal of American Ceramics Society 82(7): 1733-1740.

Lloyd JM (2004) Electrical Properties of Macro-Fiber Composite Actuators and Sensors. MS Thesis, Virginia Polytechnic Institute and State University, Blacksburg, Virginia, USA.

Ma J, Liu Y, He T, Li B and j Chu (2011) Double drive modes unimorph deformable mirror for low-cost adaptive optics. Applied Optics 50(29): 5647-5654.

Molinari G, Quack M, Arrieta AF, Morari M and Ermanni P (2014) Design and realization of a compliant adaptable wing. In: ASME 2014 Conference on Smart Materials, Adaptive Structures and Intelligent Systems: Volume 2: Mechanics and Behavior of Active Materials; Integrated System Design and Implementation; Bioinspired Smart Materials and Systems; Energy Harvesting. Newport, Rhode Island, USA.

Molinari G, Quack M, Arrieta AF, Morari M and Ermanni P (2015) Design, realization and structural testing of a compliant adaptable wing. Smart Materials and Structures 24(10).

Nelson LJ, Bowen CR, Stevens R, Cain M and Stewart M (2003) High-field behavior of piezoelectric fiber composites. In: Proceedings of the SPIE 5053, Smart Structures and Materials 2003: Active Materials: Behavior and Mechanics, volume 5053.

Nguyen M, Houwman E and Rijnders G (2017) Large piezoelectric strain with ultra-low strain hysteresis in highly $c$-axis oriented $\mathrm{pb}\left(\mathrm{zr}_{0.52} \mathrm{ti}_{0.48}\right) \mathrm{O}_{3}$ films with columnar growth on amorphous glass substrates. Scientific Reports 7(1): 1-9.

Ohanian III OJ, David B, Taylor SL, Kochersberger KB, Probst T, Gelhausen PA and Climer J (2013) Piezoelectric morphing versus servo-actuated mav control surfaces, Part II: Flight testing. In: 51st AIAA Aerosapce Sciecnes Meeting Including the New Horizons Forum and Aerospace Exposition, 20130767. Grapevine (Dallas/Ft. Worth Region), Texas, USA.

Ohanian III OJ, Hickling C, Stiltner B, Karni ED, Kochersberger KB, Probst T, Gelhausen PA and Blain AP (2012) Piezoelectric morphing versus servo-actuated mav control surfaces. In: $53 \mathrm{rd}$ AIAA/ASME/ASCE/AHS/ASC Structures, Structural Dynamics and Materials Conference. Honolulu, Hawaii, USA.

Pandey A and Arockiarajan A (2017) Fatigue study on the sensor performance of macro fiber composite (MFC): Theoretical and 
experimental approach. Composite Structures 174: 301-318.

Paradies R and Ciresa P (2009) Active wing design with integrated flight control using piezoelectric macro fiber composites. Smart Materials Structures 18: 1-9.

Park JS and Kim JH (2007) Coefficients of thermal expansion for single crystal piezoelectric fiber composites. Composites Part B: Engineering 38(7-8): 795-799.

Portela P, Camanho P, Weaver P and Bond I (2008) Analysis of morphing, multi stable structures actuated by piezoelectric patches. Computers \& Structures 86(3-5): 347-356.

Prasath SS and Arockiarajan A (2013) Effective electromechanical response of macro-fiber composite $(\mathrm{mfc})$ : Analytical and numerical models. International Journal of Mechanical Sciences 77: 98-106.

Probst TA (2012) Evaluating the Aerodynamic Performance of MFC-Actuated Morphing Wings to Control a Small UAV. PhD Thesis, Virginia Polytechnic Institute and State University, Blacksburg, Virginia, USA.

Reaves MC and Horta LG (2001) Test cases for modeling and validation of structures with piezoelectric actuators. In: 42nd AIAA/ASME/ASCE/AHS/ASC Structure and Structural Dynamics Conference. Seattle, Washington, USA.

Schrock J, Meurer T and Kugi A (2010) Control of a flexible beam actuated by macro-fiber composite patches: Ii. hysteresis and creep compensation, experimental results. Smart Materials and Structures 20(1): 015016.

Schultz MR (2003) Use of piezoelectric actuators to effect snapthrough behaviour of unsymmetric composite laminates. $\mathrm{PhD}$ Thesis, Virginia Polytechnic Institute and State University, Blacksburg, Virginia, USA.

Schultz MR and Hyer MW (2004) A morphing concept based on unsymmetric composite laminates and piezoceramic $\mathrm{mfc}$ actuators. In: 45th AIAA/ASME/ASCE/AHS/ASC Structures, Structural Dynamics \& Materials Conference. Palm Springs, California, USA.

Senba A, Ikeda $\mathrm{T}$ and Ueda $\mathrm{T}$ (2010) A two-way morphing actuation of bi-stable composites with piezoelectric fibers. In: 51st AIAA/ASME/ASCE/AHS/ASC Structures, Structural Dynamics, and Materials Conference. Orlando, Florida, USA.

Steiger K and Mokrý P (2015) Finite element analysis of the macro fiber composite actuator: macroscopic elastic and piezoelectric properties and active control thereof by means of negative capacitance shunt circuit. Smart Materials and Structures 24(025026): 1-14.

Stoney GG (1909) The tension of metallic films deposited by electrolysis. In: Proceedings of the Royal Society London Series A, volume 82. pp. 172-175.
Stuebner M, Smith RC, Hays M and Oates WS (2009) Modeling the nonlinear behavior of macro fiber composite actuators. In: Proceedings of the SPIE 7289, Behavior and Mechanics of Multifunctional Materials and Composites. pp. 728913728918.

Tan D, Yavarow P and Erturk A (2017) Nonlinear structural dynamics of macro-fiber composite cantilevers for resonant actuation. In: ASME 2017 Conference on Smart Materials, Adaptive Structures and Intelligent Systems, volume Volume 2: Modeling, Simulation and Control of Adaptive Systems; Integrated System Design and Implementation. Snowbird, Utah, USA.

Tan D, Yavarow P and Erturk A (2018) Nonlinear elastodynamics of piezoelectric macro-fiber composites with interdigitated electrodes for resonant actuation. Composite Structures 187: $137-143$.

Tawfik SA, Stefan Dancila D and Armanios E (2011) Unsymmetric composite laminates morphing via piezoelectric actuators. Composites Part A: Applied Science and Manufacturing 42(7): 748-756.

Timoshenko S (1925) Analysis of bi-metal thermostats. Journal of the Optical Society of America 11: 233-255.

Timoshenko S and Woinowsky-Kreiger S (1959) Theory of Plates and Shells. New York: McGraw Hill.

Weinberg M (1999) Working equations for piezoelectric actuators and sensors. Journal of Microelectromechanical Systems 8(4): 529-533.

Wilkie W, Inman D, High J and Williams R (2004) Recent developments in NASA piezocomposite actuator technology. In: 9th International Conference on New Actuators. Breman, Germany.

Williams BR, Park G, Inman DJ and Wilkie WK (2002) An overview of composite actuators with piezoceramic fibers. In: Proceedings of 20th International Modal Analysis Conference, volume 4753. Los Angeles, California, USA, pp. 421-427.

Williams RB (2004) Nonlinear Mechanical and Actuation Characterization of Piezoceramic Fiber Composites. $\mathrm{PhD}$ Thesis, Virginia Polytechnic Institute and State University, Blacksburg, Virginia, USA.

Williams RB, Inman DJ and Wilkie WK (2006) Nonlinear response of the macro fiber composite actuator to monotonically increasing excitation voltage. Journal of Intelligent Material Systems and Structures 17(7): 601-608.

Williams RB, Schultz MR, Hyer MW, Inman DJ and Wilkie WK (2004) Nonlinear tensile and shear behavior of macro fiber composite actuators. Journal of Composite Materials 38(10): 855-869. 
Xu X, Li B, y Feng and Chu J (2007) Design, fabrication and characterization of a bulk- pzt-actuated mems deformable mirror. Journal of Micromechanics and Microengineering 17: 2439-2446.

Zhang SQ, Li YX and Schmidt R (2015) Modeling and simulation of macro-fiber composite layered smart structures. Composite Structures 126: 89-100.

Zheng X, Lin L, Ichchou MN and Chao L (2017) Hysteresis and the nonlinear equivalent piezoelectric coefficient of $\mathrm{mfcs}$ for actuation. Chinese Journal of Aeronautics 30(1): 88-98. 